\title{
On the plasma confinement by acoustic resonance
}

\section{An innovation for electrodeless high-pressure discharge lamps}

\author{
Gilles Courret, Petri Nikkola, Sébastien Wasterlain, Olexandr Gudozhnik, Michel Girardin, Jonathan Braun,
} Serge Gavin, Mirko Croci, and Peter W. Egolf

Department of Industrial Technologies, University of Applied Sciences and Arts Western Switzerland (HES-SO), Yverdon-lesBains, 1401 Switzerland (see http://www.heig-vd.ch/rad/mnt). e-mail: gilles.courret@heig-vd.ch

Received: date / Revised version: date

\begin{abstract}
In an applied research project on the development of a pulsed microwave sulfur lamp prototype of $1 \mathrm{~kW}$, we have discovered an amazing phenomenon in which the plasma forms a ball staying at the center of the bulb despite gravity, thus protecting the glass from melting. In this paper, it is shown that this results from an acoustic resonance in a spherical mode. Measurements of the plasma response to short pulses are presented showing beats at the spherical resonance. It is demonstrated that the beats could result from the simultaneous excitation of two normal modes with a frequency difference of approximately $1 \%$. One of the two frequencies matches precisely the microwave pulses repetition, a little below $30 \mathrm{kHz}$. Thus this one is due to a forced oscillation, whereas the other one is due to a free oscillation. The phase velocity of sound was calculated as a function of temperature in order to find the series of temperatures at which a resonance would occur if the bulb were an isothermal solid sphere. The mean temperature inside the actual bulb was determined from the only doublet of this series, that has characteristic frequencies close enough to cause the observed beats. In addition, one of these two modes has a spherical symmetry that can explain the plasma ball formation. The obtained mean temperature is consistent with the direct measurements on the bulb surface as well as with the temperature in the core of a similar plasma found in the literature. We have also proposed a model of the resonance onset based on the acoustic dispersion and the sound amplification due to electromagnetic coupling.
\end{abstract}

Key words. molecular plasma - pulsed microwave plasma - plasma confinement - acoustic resonance acoustic dispersion - sound amplification due to electromagnetic coupling - plasma of sulfur - electrodeless light bulb - high-pressure lamps - light sources

\section{Introduction}

In the frame of an applied research project on a pulsed microwave sulfur lamp, we have discovered a phenomenon of resonance in which the plasma forms a ball centered in the electrodeless bulb. This publication aims to report this discovery and to give a first interpretation of it. The setup has not been designed to investigate the physics of this phenomenon, but rather to build a demonstrator of this lamp. However, we have developed a physical model to give an interpretation of the observations, calculate the average temperature in the bulb from the measurement of the resonance frequency and ease the resonance search as this phenomenon is very furtive. Our experiments of heating interruption have in addition shown that the molecular dissociation can be governed by the pure vibrational mechanisms [1].

The light bulb is filled with a small quantity of sulfur, which is in solid state at room temperature, and argon

Send offprint requests to: gas at low pressure in order to ignite the lamp by the electrical breakdown of the gas. The warm-up is obtained as the sulfur evaporates, producing a weakly ionized vapor which efficiently absorbs the microwaves. Indeed, the vibrational excitation of the polyatomic molecules might enhance liberation of electrons by a stepwise ionization [2]. The higher the temperature, the higher the vibrational excitation. The resulting additional ionization contributes to raise the microwave absorption since it is due to the collisions of free electrons with molecules. The microwave absorption is then an increasing function of temperature as well as of pressure, as observed during the warm-up phase. In the operational conditions the main chemical reaction is $\mathrm{S}_{2} \leftrightarrow 2 \mathrm{~S}[3]$. The sulfur recombination produces diatoms in an electronic excited state of radiative de-excitation $2 \mathrm{~S} \rightarrow \mathrm{S}_{2}^{*} \rightarrow \mathrm{S}_{2}+\gamma$ [4]. This is the main mechanism of the light emission [5].

The microwaves are produced with a $2.45 \mathrm{GHz}$ standard magnetron whose emission is confined in a cylindrical grid surrounding the spherical quartz bulb. The lamp Solar-1000 working on the same principle, which in the 
1990s has been commercialized by an American company [6], has a wall-plug efficiency that overpasses $110 \mathrm{Lm} / \mathrm{W}$ at the full input power of $1.4 \mathrm{~kW}$. Because the microwave absorption is increasing with temperature, at least in the operating conditions, a thermal avalanche occurs, resulting in the bulb fusion. Therefore, presently commercialized sulfur lamps need to be equipped with a bulb being in permanent rotation. The rotational motion creates convection cells inside the bulb, so that plasma from the hot central part is constantly mixed with colder gas from the peripheral zone, close to the inner surface of the bulb [7]. However, the requirement of a constant rotation is a major commercial drawback, since it requires an electric motor in permanent operation.

Researchers at University of Applied Sciences and Arts Western Switzerland (HES-SO) have found a novel way to suppress the rotating motion [8]. If the microwave flux is modulated in the radio frequency range $(\mathrm{kHz})$, ultrasonic waves are produced inside the bulb and, at acoustic resonance, the plasma stabilizes. Moreover, under certain conditions the resonance can confine the plasma into a sphere in the center of the bulb, which is also spherical, see Fig. 1. The heating is then enough centralized to prevent the bulb from fusing. In addition the light emission is isotropic. Since the impinging microwaves can be described as plane waves, the $\mathbf{E} \times \mathbf{B}$ drift pushes the plasma, so that it forms a flame unless the duty-cycle is reduced. Even then the asymmetry tends to prevent the excitation of the spherically symmetric acoustic waves. The free convection also reduces the symmetry. In absence of modulation, the brightest part of the plasma settles down in the upper part of the bulb, because of the density gradient, but in the opposite case, when the modulation is well tuned it settles down in the central part [9]. This sustentation effect is due to the ultrasonic wave produced by the modulation. In that condition, the oscillation of pressure is the greatest in the centre of the bulb. Hence, if the microwave pulses are released at the moments the pressure in the centre reaches its maximum, most of the delivered energy is absorbed there, because the microwave absorption is an increasing function of pressure. In this way, a standing acoustic wave is driven. Furthermore, since most of the heat is released in the centre, the risk of melting the bulb is much reduced. This allows, therefore, increasing greatly the maximum power a bulb can admit, as well as the luminous efficiency as a result [10].

The paper is organized as follows: The experimental setup is briefly described in Sect. 2 and measurements of the plasma dynamics are presented in Sect. 3, showing a transformation at resonance that gives rise to beats. In order to determine the mean temperautre in the bulb, in Sect. 4 we calculate the velocitiy of sound in the plasma as a function of its temperature by means of a thermodynamic model that notably allows us to take into account dispersion. Sect. 5 examines the electromagnetic coupling of sound, because the interplay of acoustic oscillations with the electromagnetic induction governs the resonance onset. In Sect. 6, we propose an interpretation of our discovery which gives us a way to measure the mean temperature by using the fact that there is only one possible pair of normal modes of oscillation that can cause the observed beats. Finally we are able to assess the mean plasma characteristics and discuss the optimization of the luminous efficiency, the stability of the spherical resonance and how it onsets.

\section{Experimental setup}

The experimental installation constitutes of the main parts shown in Fig. 2. The magnetron is a $2.45 \mathrm{GHz}$ domestic microwave source with an added RLC anti reflection filter. The high tension unit, feeding the magnetron, has been designed and constructed in-house [11]. It is controlled by a high frequency pulse generator, whose design is based on a digital signal processor (DSP). Pulses can be produced with the tension of $4.5 \mathrm{kV}$, having a modulation frequency up to $70 \mathrm{kHz}$ and a duty-cycle down to about $10 \%$. The photo-sensor is of the type Hamamatsu C6386-01, whose bandwidth was set to $10 \mathrm{MHz}$. They are connected to a $\mathrm{PC}$ with data acquisition cards with a maximum acquisition frequency above $60 \times 10^{6}$ samples/s. The samples are averaged by blocks, lowering the sampling frequency, in order to improve the signal-to-noise ratio. The installation is computer controlled via Matlab, enabling the operator to change the modulation frequency and the duty-cycle on the fly. The PC is connected to the pulse generator with a RS232 connection. In addition, the bulb surface temperature is monitored with a high temperature infrared camera. The bulb is a hollow sphere, made of pure silica, with an inside volume of $15.6 \mathrm{~cm}^{3}$. It is filled with argon gas and an amount of sulfur having a concentration of $1.8 \mathrm{mg} / \mathrm{cm}^{3}$. The argon content is much smaller than that of sulfur, because the argon pressure at ambient temperature is tens of millibars. The bulb is enclosed inside a metal mesh which acts as a Faraday cage, preventing any harmful leak of the microwave field.

\section{Experimental results}

\subsection{Observations}

In order to qualitatively appreciate the optical thickness of the plasma, we have installed a helium-neon laser of $15 \mathrm{~mW}$ of emitted power, pointing to the center of the bulb. The principle is to observe the red brightness of the laser spot, projected onto a white diffusive screen, placed behind the bulb. It was observed that the spot declines progressively during the warm-up phase. The optical thickness therefore increases extensively during the evaporation of sulfur, as it is expected, since the number density of the molecules in the vapor becomes much larger. When the steady regime is reached, the laser red spot cannot be distinguished anymore on the screen strongly illuminated by the white light coming from the plasma, confirming that its core in operating condition is optically thick (optical depth is about $10 \mu \mathrm{m}$ according to [12]). In 
contrast, the vapor around the plasma core remains transparent, since one can see the rear grid through it.

Moreover, we have observed the motions of the plasma with an ultra fast camera (Vision Research Phantom) filming in color at $10^{5} \mathrm{fps}$ with a resolution of $256 \times 256$ (see Fig. 3). After the warm-up phase, the electromagnetic coupling becomes much stronger [13]. If no acoustic resonance stabilizes the plasma, it shows stochastic shakings. To the human eye, the light is vibrating, meaning that the vibration spectrum goes below $70 \mathrm{~Hz}$, the upper bound of the human vision bandwidth. We have, furthermore, observed the plasma evolution in smaller time scales by viewing the film in slow motion. Neither its shape nor the brownish or bluish color of its sheath appears to vary in the duration of a modulation period $(\approx 40 \mu s)$. The only events visible at this time scale were plasma filaments usually located along the bulb wall (see Fig. 3) when the plasma was not in spherical resonance. The filaments were blinking at the modulation frequency of the magnetron, in concordance with the pulse duty-cycle; they were certainly occurring when the magnetron was switched on. The occurrence of these electric arcs, close to the bulb wall, seems to confirm the importance of the stepwise ionization in the electromagnetic coupling of the plasma. Indeed, the vibrational relaxation can be slow with respect to the microwave modulation, especially in the cold zones according to (2) (see Sect. 3.2). And as a result, the convection might produce currents of high vibrational temperature streaming out of the plasma core. Stepwise ionization might enrich them with free electrons so much that paths of electrical current constriction appear.

Furthermore, we note that in all the experiments where the plasma ball has been formed, its size was always about half the bulb size (see Fig. 1). This feature can be explained by acoustic reflections on the bulb wall, since the magnetron switching modulates the pressure. When energy is supplied and the pressure is locally peaking at some point, called source point, a large amount of heat is released there, because the collision frequency of free electrons into the molecules is a growing function of pressure. As a result, from each of the source points an acoustic wave is emitted. The question is: Under which conditions the reflections on the bulb wall may produce new source points at next pulses? There is of course a matter of synchronization, as confirmed by experiments (see Fig. 18, Sect. 6.6). However, acoustic reflections lead also to geometric conditions, which are now analyzed.

We consider the acoustic emission towards the closest wall to the source point separately from the emission towards the opposite wall (see Fig. 4). These spherical domes form two concave mirrors facing each other, each having a focus point laying at half of the bulb radius $(a / 2)$ symmetrically to the other, in the Gauss approximation. It is well-known that the image of a source point is real only if its distance to the mirror is greater than the focal distance. Otherwise, the image is virtual and the absence of a pressure peak prevents this wave from regaining energy during the next microwave inflow. Assuming the gas is homogeneous and non dispersive, we assess the image position using of the Spherical Mirror Equation,

$$
\frac{1}{y}+\frac{1}{y^{\prime}}=\frac{2}{a}
$$

where $y$ is the source distance and $y^{\prime}$ its image distance to the mirror. If the source point lies in a centered sphere of half radius, $y>a / 2$ for both mirrors, both of its images will be real, $y^{\prime}>0$, and thus the wave will be fully recharged at the next pulse. On the contrary, if the source point lies out of the sphere with half-radius, it will have only one real image, the one made by the reflection in the furthest mirror, i.e. in the case $3 a / 2 \leq y \leq 2 a$. Then, according to (1), $2 a / 3 \leq y^{\prime} \leq 3 a / 4$, giving $a / 2 \leq y^{\prime}$. Hence, this real image is lying inside the sphere with half-radius. So virtual images put apart, the secondary image of the sphere with half-radius is real and included in itself, allowing to localize the heat release to this spatial domain. This is certainly the reason why the plasma ball expands to half of the bulb radius.

\subsection{Plasma dynamics measurements}

Fig. 5(a) shows the AC component of the photo signal of the plasma, which is not in spherical resonance. The AC component is the signal minus the DC component, i.e., its mean value during one cycle. In this experiment the magnetron modulation frequency was $f_{c}=26.1 \mathrm{kHz}$ and the duty-cycle was $24.7 \%$. The modulation frequency is defined as $f_{c}=1 / T_{c}$, where $T_{c}$ is the duration of one modulation cycle, as shown in Fig. 5(a), and the corresponding angular frequency is $\omega_{c}=2 \pi f_{c}$. As can be seen, the photo signal has a regular triangular shape, and on each modulation period the signal consists of two distinct parts, as shown below.

During the discharge, i.e. in the switched-on periods as shown by the gray line, which is the normalized amplitude command signal, the photo signal usually increases linearly. The rise rate, normalized to the DC value of the tension, is in this example about $10^{4} \mathrm{~s}^{-1}$. Because of the applied electromagnetic field, the ionization may locally reach high peak values. It is known that a large fraction of electron energy from non-thermal discharges in several gases goes mostly into the vibrational excitation at electron temperatures ranging from $1 \mathrm{eV}$ to $3 \mathrm{eV}$ [14]. Indeed, the vibrational excitation occurs in such plasmas at much lower electron energies than required by the electronic excitation $(>10 \mathrm{eV})$ [15]. So the vibrational energy may reach high values at ionization peaks [16]. The vibrational excitation by electron impact may occur over the whole vibrational ladder, through resonant interaction, like in the case of the pulsed discharges in atmospheric nitrogen studied by Colonna et al. [17]. Besides a preceding study of the lamp has shown that the pulsed mode of microwave induction increases strongly the mean light flux in comparison to the continuous mode [10]. So, as a matter of fact the recombination rate is higher in the pulsed mode and, since the upper electronic energy level is fed by the recombination process, it appears that the pulses 
pump the vibrational excitation. In situations as shown in Fig. 5, where the light flux is much modulated, the dissociation - recombination kinetics is certainly affected by the pulse mode. The amplitude of the photo signal modulation is however changing, as one can observe on Fig. 7; this is probably due to the instability of the vibrational relaxation.

At the turn-off, the electron temperature drops faster than the temperature of the neutral component [18]. The dissociation rate from the pure vibrational mechanisms, in which only heavy particle collisions are involved, may become competitive with the direct electron impact dissociation mechanisms, as in the case study of Pietanza et al. on a $\mathrm{CO}_{2}$ microwave plasma, based on a model of the coupling of the electron energy distribution with the vibrational kinetics [19]. The afterglow decay might be very fast, as long as the electron temperature lays above the vibrational temperature, as it has been found by Capitelli et al., also in numerical modelizations of a $\mathrm{CO}_{2}$ plasma [20]. In a model of the triangular response to short microwave pulses, the decay is composed of two successive exponential parts:

- First a fast exponential decay occurs. As long as the gas is still enriched in highly excited or dissociated molecules, the vibrational relaxation might be accelerated as a result of the elevation of the vibrational ditribution as well as of the chemical interactions between colliding reactants [21]. In this part of the decay process, seen as the remanence of the electromagnetic coupling, the characteristic relaxation time is $\tau_{e}$.

- In the subsequent period of time, the vibrational kinetics might be governed by the superelastic collisions with both vibrational and electronic excited states, as derived by D'Ammando et al. [22] and analysed by Colonna et al. in the case of high pressure hydrogen plasmas [23]. As a matter of fact, the relaxation time lengthens often; a second characteristic value $\tau_{v}$ is measured.

Johnston et al. have measured the characteristic decay time of the electron temperature to be less than $10 \mathrm{~ns}$ [24]. The experimental conditions such as the bulb size and its content, the microwave frequency and the mean power, were similar in our experiments. They used a continuous plasma heating and a rotating bulb. In our lamp, in contrast, the energy is supplied to the plasma by short pulses. As the degree of ionization peaks locally during such pulses, the vibrational relaxation is temporarily fast, which leads to the characteristic time $\tau_{e}$

The photo signal of the spherical resonance plasma is shown in Fig. 6(a). In this experiment the duty-cycle was $12 \%$ and $f_{c}=28.2 \mathrm{kHz}$. The photo signal is almost a perfect sinusoid. In addition, a phase shift appears between the magnetron command signal, shown in gray in normalized units, and the photo signal. In this sampling, the plasma brightness is at maximum when the magnetron power is switched off. This shows that the molecular dissociation is then governed by the pure vibrational mechanisms, i.e. by the collisions between vibrationally ex- cited molecules, dominating the electron impact dissociation mechanisms like in the study of Capitelli et al. on other molecular plasmas [25]. The behavior is different from the non-spherical case of Fig. 5(a) in two main aspects. First, the photo signal shape is different; second, the photo signal and the command signal are not in the same phase. In Fig. 6(a), the definition of the frequency of the photo signal is also shown. The period of oscillations is $T_{p}$ and the frequency is $f_{p}=1 / T_{p}$. The angular frequency of the photo signal is $\omega_{p}=2 \pi f_{p}$. Fig. $6(\mathrm{~b})$ shows the photo signal over a period of $50 \mathrm{~ms}$. In gray is shown a beating signal, which is the sum of two sine waves differing $1 \%$ in frequency, i.e. $\sin \left(2 \pi f_{c} t\right)+\sin \left(2 \pi\left(f_{c}-\Delta f\right) t\right)$ with $\Delta f=282 \mathrm{~Hz}$. As one can see, this sum of two sine waves produces a similar beat as in the measured photo signal. As a matter of fact, the photo signal spectrum, obtained by Fourier transform of $50 \mathrm{~ms}$ samples, shows several peaks in the neighborhood of the pulse repetition frequency. The spectrum of the photo signal of Fig. 6(b) is presented in Fig. 6(c). Clearly two dominating modes exist, one is exactly at $f_{c}$, while the other is at $0.991 f_{c}$. With $f_{c}=28.2 \mathrm{kHz}$ the separation of the peaks is $250 \mathrm{~Hz}$, which is equal to the beat frequency up to the measurement accuracy. We, therefore, make a hypothesis that in the spherical resonance there are actually two normal modes simultaneously excited with a frequency difference of about $250 \mathrm{~Hz}$ or $1 \%$.

The transition of two plasmas into the spherical resonance is shown in Fig. 7. In Fig. 7(a) is the AC component of the photo signal. The signals are horizontally shifted such that the transitions are at $t=17 \mathrm{~ms}$ for both plasmas. At this moment the amplitude decreases during only one period from $220 \mathrm{mV}$ to $70 \mathrm{mV}$ and from $86 \mathrm{mV}$ to $30 \mathrm{mV}$ for the plasma marked with the gray and black line, respectively. The transition is accompanied with a sudden increase of the characteristic time $\tau_{e}$. The product $\tau_{e} \omega_{p}$ is shown in Fig. 7(b). When the plasma is not in the spherical resonance, this product is typically ranging between 0.5 and 3, as one can also see in Fig. 5(b). However, it increases in the transition; values above 4 have been measured. In the spherical resonance, the response becomes sinusoidal and no exponential decay can be found anymore. But, from the trends observed at the start of the resonance, it seems that $\tau_{e} \omega_{p}$ rises much above 5 as one can see in Fig. 7(b). Moreover, as shown by Fig. 7(c), the vibrational relaxation time $\tau_{v}$ also undergoes a sharp rise at the very moment the amplitude decreases. Nevertheless, it does not rise much above the values it showed long before the transition. In these experiments, the resonance was not stable and the response fell back into the triangular type after about $6 \mathrm{~ms}$.

Tests of heating interruption have also been performed, in where the microwave pulses are stopped for a number of periods, as shown in Fig. 8. The DC component of the photo signal decreases with a time constant of about $9 \mathrm{~ms}$ for all plasmas; the measurements of Fig. 8 give $8.7 \pm 0.5 \mathrm{~ms}$ in the case 'in resonance' and $8.6 \pm 0.5 \mathrm{~ms}$ in the opposite case. Comparing with the measurements of $\tau_{v}$, which vary in the range of $1 \mu \mathrm{s}-30 \mu \mathrm{s}$ in the cases 
of Fig. 5(b) and Fig. 7(c), we observe that the characteristic time of the exponential decay model increases as the plasma cools down. This is in qualitative agreement with the general theory of chemical and vibrational nonequilibrium. Indeed, molecular thermal relaxation is due to collisions between molecules. A much larger number of collisions is required for vibrational relaxation than for the other degrees of freedom. The required number of collisions depends moreover on the relative kinetic energy between the two colliding particles; the lower the temperature, the greater is the number of required collisions for vibrational energy exchange. Indeed for most diatomic gases, $\tau_{v}$ is a decreasing function of the translational temperature $T$ (simply called 'temperature'), at a given pressure $p$, as shown by the Landau-Teller rate model [26]

$$
p \tau_{v}=C_{1} \exp \left[\left(\frac{C_{2}}{T}\right)^{1 / 3}\right]
$$

where $C_{1}$ and $C_{2}$ are two physical constants of the gas. Although this model does not take into account the vibration - vibration energy transfer as well as the anharmonic pumping [27], it is used as a first approximation for most polyatomic gases in vibrational nonequilibrium [28], admitting three basic assumptions [29]: (a) the transitions occur only between the neighboring vibrational states, (b) the transition rates are proportional to the vibrational quantum number, and (c) the levels are populated according to a Boltzmann distribution. Solving numerically the master equations for nonequilibrium distribution functions is out of scope in the present work, but we refer to (2) in qualitative discussions.

Furthermore, according to kinetic theory, the collision frequency is proportional to pressure and inversely proportional to the square root of temperature. Those two parameters are bound in our case, because the gas volume is constant, and the collision frequency decreases when the plasma cools down. This rarefaction of the collisions is still enhanced by the recombination, which reduces the number density of particles, as well as by the condensation. In addition, the required number of collisions increases as temperature and pressure decreases, see (2). Each of these effects lengthens the characteristic time of the vibrational relaxation. Lastly, the heat of recombination and condensation is progressively released back, which slows down the decrease of temperature. In this respect, our values of decay time are not inconsistent with the ones measured in [24], laying around $20 \mathrm{~ms}$, thus significatly above ours, as the switch-off period in [24] was longer.

The tests of long power interruption also allowed us to measure the acoustic quality factor of the bulb, $Q$. If the plasma is initially in the spherical resonance, the sinusoidal AC component of the photo signal attenuates very slowly, see Fig. 8 (a). In contrast, the beats mentioned above disappear immediately after the power cut-off, see Fig. 8 (b). Therefore, it seems that one of the two normal modes is forced and, consequently, its frequency is equal to $f_{c}$, whereas the other is a free oscillation. In order to determine the quality factor in the remaining freely oscillating mode, we have measured the decrease of the AC amplitude of the photo signal. Assuming an exponential decay $\Delta s(t)=\Delta s_{0} e^{-t / \tau_{a}}$ where $\Delta s_{0}$ is the initial amplitude, see Fig. 6(a) for the amplitude definition, and measuring the amplitude after $n$ oscillations, $\Delta s_{n}$, the attenuation time is given as $\tau_{a}=\Delta t / \ln \left(\Delta s_{0} / \Delta s_{n}\right)$. The quality factor is then

$$
Q=\frac{\omega_{p} \tau_{a}}{2}=\frac{n \pi}{\ln \left(\Delta s_{0} / \Delta s_{n}\right)}
$$

with $\omega_{p}=2 \pi n / \Delta t$. We have measured the quality factor in several experiments. When the plasma was in spherical resonance, we found a value $Q=500 \pm 100$ which corresponds to the attenuation time of $\tau_{a}=(6 \pm 1) \mathrm{ms}$. In contrast, we could not make any conclusive measurement when the plasma was not in the spherical resonance. So it appears that, in this case, the electron impact dissociation mechanisms have a determinant effect, in contrast to the opposite case, where it is the pure vibrational mechanisms that are governing dissociation, like in the case study [20].

\section{Velocity of sound}

A model is developed for the phase velocity of sound, taking into consideration the dispersion due to vibrational relaxation. The temperature dependence of the velocity then allows us to find the gas temperature in the resonance.

During each microwave pulse, the degree of ionization may momentarily and locally rise, but the fraction of charged particles remains low. In a weakly ionized gas, the collisions involving at least one neutral particle are so much dominating, that the ions and the electrons are forced to follow the neutrals in the acoustic oscillations [30]. In this respect, we assume that the sound propagates like in a non ionized vapor. In addition the ideal gas model is valid since the pressure in the bulb is in the range of 3 to 6 bar [24], far below 182.1 bar, which is the critical pressure of sulfur vapor [31]. In the present work, we have moreover adopted a one-node-lumped model as the objective is the interpretation of the photo signal variations in time, i.e., the global response of the plasma.

\subsection{Thermodynamic basis}

Measurements of the dissociation energy of disulfur by photoionization and thermochemistry give the same value of $4.38 \mathrm{eV}$ [32]. We use $D=4.38 \mathrm{eV}$ from the zero-point energy of vibrations (cf. Fig. 22). Regarding ionization, its energy is more than twice as high, $9.36 \mathrm{eV}$, according to [32]. As a result, the main chemical reactions in operating condition, that occur inside the bulb, are the dissociation and the recombination of disulfur. Taking into account this only chemical cycle, we consider a collection of molecules, composed of three chemical species:

1. $N_{A}$ atoms of argon $\mathrm{Ar}$, of molar mass $M_{A}$,

2. $N_{1}$ atoms of sulfur $\mathrm{S}_{1}$, of molar mass $M_{S}$,

3. $N_{2}$ diatoms of sulfur $\mathrm{S}_{2}$, of molar mass $2 M_{S}$. 
The volume taken by the gas $\left(15.6 \mathrm{~cm}^{3}\right)$ and the enclosed mass are given constants. The contribution of argon to the total mass density, $\rho=\rho_{A}+\rho_{S}$, is in the magnitude order of one percent. The content in Sulfur is $\rho_{S}=1.82 \pm$ $0.02 \mathrm{~kg} / \mathrm{m}^{3}$.

Furthermore, if no dissociation occurred, the number of sulfur diatoms would be constant and equal to $N_{0}=$ $N_{2}+N_{1} / 2$. The proportion between the two sulfur chemical species is given by the degree of dissociation $\alpha$

$$
N_{2}=N_{0}(1-\alpha) \text { and } N_{1}=2 N_{0} \alpha .
$$

As a result the mean molecular mass is a function of $\alpha$

$$
\bar{M}=\frac{N_{A} M_{A}+2 N_{0} M_{S}}{N_{A}+N_{0}(1+\alpha)} .
$$

Besides we assume that the sound wave process is adiabatic as the quality factor of the spherical resonance is high (see Sect. 3). The Laplace relation gives, hence, the phase velocity of sound in an ideal gas of mass density $\rho$ at pressure $p$ or translational temperature $T$, which is simply called 'temperature'. ( $T$ is homogeneous to energy in order to lighten the algebraic notation)

$$
v^{2}=\gamma \frac{p}{\rho}=\gamma \frac{T}{\bar{M}}
$$

where $\gamma$ is the isentropic coefficient, defined as the following partial derivative at constant entropy [33]

$$
\gamma=\left(\frac{\partial \ln (p)}{\partial \ln (\rho)}\right)_{S}
$$

This parameter can be expressed as a function of the degree of dissociation, $\alpha$, and the isobaric and isochoric heat capacities per unit mass, respectively $c_{p}$ and $c_{v}$

$$
\gamma=Z_{d} \frac{c_{p}}{c_{v}}
$$

where

$$
Z_{d}=\frac{2}{(1+\alpha)(2-\alpha)}
$$

is the correction factor due to dissociation/recombination, which is lying between 1 and $8 / 9$, i.e. its value at halfdissociation $\alpha=0.5$ [34]. In the case of a non-reacting gas $Z_{d}=1$.

\subsection{Dispersion}

We note that the Laplace relation (6) shows no dependence on the sound frequency. However, dispersion is negligible only if the thermal changes, which take place as the gas is submitted to compression/expansion cycles, occur so slowly that the changes in the energy of all the degrees of freedom of the molecules follow with no significant time lag. For the vibrational energy in the operational frequency range, $20 \mathrm{kHz}-70 \mathrm{kHz}$, where the critical time is $2 \mu \mathrm{s}-8 \mu \mathrm{s}$ and the measured relaxation times are also of the order of microseconds (see Fig. 7), this is certainly not true. Therefore, in order to take the dispersion into account, we must regard the heat capacity as a complex function of frequency, the imaginary part corresponding to the phase difference between the pressure and density fluctuations. As this outphasing is small in cases of practical importance we have, to a good approximation, $\operatorname{Re}[\sqrt{\gamma}] \approx \sqrt{\operatorname{Re}[\gamma]}[35]$. So, to obtain the sound velocity $v$, i.e. the real part of the complex sound velocity, the Laplace relation (6) is rewritten as follows

$$
v=\sqrt{\operatorname{Re}[\gamma] \frac{p}{\rho}}
$$

where the coefficient $\gamma$ is now the following complex number

$$
\gamma=Z_{d}+\frac{1}{\bar{M}} \frac{Z_{d}+i \omega \tau_{v}\left(1-\bar{M} c_{\infty}\left(Z_{d}-1\right)\right)}{c_{0}^{\star}+i \omega \tau_{v} c_{\infty}},
$$

where $c_{\infty}$ is the limit of $c_{v}$ when $\omega \rightarrow \infty$. The two limits, at low and high frequency, are labeled as equilibrium and frozen, respectively. Hence, the equilibrium isentropic coefficient is given by the low frequency limit of (11)

$$
\gamma_{0}=Z_{d}\left(1+\frac{1}{\bar{M} c_{0}^{\star}}\right) .
$$

The factor $Z_{d}$ holds for the coupling between the chemical reaction and the vibrational relaxation. The molecules that are lying in high vibrational energy levels are indeed more readily dissociated. But if the vibrational degree of freedom is frozen, the coupling has no impact on the sound propagation. And indeed, $Z_{d}$ vanishes at the high frequency limit of (11), see (21).

By matching (12) with (8), we get the effective isochoric equilibrium heat capacity per unit of mass $c_{0}^{\star}$ by

$$
1+\frac{1}{\bar{M} c_{0}^{\star}}=\frac{c_{p}}{c_{v}}
$$

This ratio is derived in Appendix A, see (67), giving hence

$$
c_{0}^{\star}=c_{0} \frac{2+3 \alpha}{2+\alpha\left(7 / 2-D^{\star} / T\right)},
$$

where $c_{0}$ is the equilibrium value of $c_{v}$; this parameter is derived in Appendix B.

\subsection{Degree of dissociation}

It is known that vibrational pumping can enhance the dissociation rate, but to take this dynamics into account, it would be necessary to solve numerically the Boltzmann equation for the electron energy distribution function with nonequilibrium vibrational kinetics [19]. In the present study, we assume that the degree of dissociation is in the average governed by the mean temperature in the bulb. We use the classical equilibrium thermodynamics, with the equilibrium constant, as defined in Appendix A by 
(59). We have first assessed it by the following expression [36]

$$
K_{e}(T)=p^{0} \exp \left(\Delta S_{0}-\frac{D}{T}\right),
$$

where $\Delta S_{0}$ is the molar entropy of dissociation under the standard pressure, $p^{0}=10^{5} \mathrm{~Pa}$. According to our calculations, which are based on a thermodynamic properties data base [37], (15) leads to the values of the equilibrium constant of dissociation shown in Fig. 9.

For comparison, we have also calculated $K_{e}(T)$, using the expression given in [38],

$$
K_{e}(T)=C^{s t} \sqrt{T} \exp \left(-\frac{D}{T}\right) .
$$

We have determined the constant of this expression, $C^{s t}=$ $9.9513 \times 10^{20} \mathrm{~Pa} \mathrm{~J}^{-1 / 2}$, by fitting published data of a 1 $\mathrm{kW}$ microwave sulfur lamp, calculated by applying a onedimensional local thermodynamic equilibrium model [39]. Even if in that study the magnetron was in the continuous mode, the luminous efficiency is close to values measured in our system [40], as the bulb was rotating in this lamp. The fit should therefore allow us to approach the effective DC value, reached in the operating conditions of our lamp. As shown in Fig. 9, the two curves are almost superposed and, therefore, the two models are in agreement. From now on we use (16), because the derivative in respect to temperature will be required.

Furthermore introducing the ideal gas law and (4) in the definition of $K_{e}$ (see (59)) leads to

$$
K_{e}(T)=\frac{4 \alpha^{2}}{1-\alpha} n_{0} T
$$

The degree of dissociation is then found as the solution of

$$
4 n_{0} T \alpha^{2} K_{e}(T)^{-1}+\alpha-1=0
$$

with the positive solution being

$$
\alpha=\frac{2}{1+\sqrt{1+16 n_{0} T / K_{e}(T)}} .
$$

Figure 10 shows the obtained dissociation versus temperature in the present enclosed vapor $\left(n_{0}=1.69 \times 10^{19} \mathrm{~cm}^{-3}\right)$.

\subsection{Frozen phase velocity of sound}

Ultrasounds are produced inside the bulb by the magnetron switching. The acoustic intensity certainly peaks at the turn-off as the variation of electromagnetic induction is then the most abrupt [41]. Hence, the relevant characteristic time for the acoustic resonance onset is $\tau_{e}$, according to our analyse of the transition from triangular to sinusoidal response (Sect. 3.2). In addition, $\omega \tau_{e}<3$, when the plasma is not in spherical resonance, but $\omega \tau_{e}>5$, when the resonance is at onset (see Fig. 7(b)). In a first approach of the resonance conditions, we therefore approximate the phase velocity of sound by its frozen value given by the high frequency limit of (10)

$$
v_{\infty}=\sqrt{\gamma_{\infty} \frac{p}{\rho}}=\sqrt{\gamma_{\infty} \frac{T}{\bar{M}}},
$$

where $\gamma_{\infty}$ is the frozen isentropic coefficient given by the high frequency limit of (11)

$$
\gamma_{\infty}=1+\frac{1}{\bar{M} c_{\infty}}
$$

In addition, neglecting the electronic degrees of freedom, the frozen mass isochoric heat capacity of sulfur is approximated as [42]

$$
c_{S \infty} \approx \frac{7-\alpha}{4 M_{S}}
$$

whereas the corresponding heat capacity of argon is $c_{A}=$ $3 /\left(2 M_{A}\right)$. There is indeed no electronic excitation significantly involved for argon nor for atomic sulfur, since none of their emission lines peaks out of the spectrum. Moreover using (5), we obtain the following approximation

$$
\bar{M} c_{\infty} \approx \frac{7-\alpha}{2(1+\alpha)}+\frac{3 N_{A}}{2 N_{0}(1+\alpha)},
$$

which gives

$$
\frac{1}{\bar{M} c_{\infty}} \approx \frac{2(1+\alpha)}{7-\alpha+3 N_{A} / N_{0}} .
$$

Finally, substituting this result into (21), we get

$$
\gamma_{\infty} \approx 1+\frac{2(1+\alpha)}{7-\alpha+3 N_{A} / N_{0}} .
$$

Combining this result with (19) and (5), we obtain from (20) the phase velocity of sound, approximated by its frozen value, as a function of temperature (see Fig. 11).

\subsection{Volume average of the phase velocity of sound}

From the preceding analysis, illustrated in Fig. 4, we see that a stationary wave would preferably build up, if the reflections in the closest and the farthest parts of the bulb wall are synchronized, i.e. they reach their image points at the same time, during the magnetron is switched on, because otherwise they would not regain energy. We now look more closely at this issue, in considering again the plasma ball formation, see Fig. 1.

The part of the wave emitted towards the closest wall is propagating first through the cold layers of the periphery, where the degree of dissociation $(\alpha)$ is lower than in the average. In contrast, the part of the wave emitted towards the farthest wall is propagating first through the hot core, where $\alpha$ is much higher since this is occurring when the magnetron is switched on. As seen in Sect. 4.4, the frozen velocity of sound is a growing function of temperature, see (20). Moreover, the mean molar mass $(\bar{M})$ 
decreases when $\alpha$ increases, see (5), adding to the acceleration of the wave. Furthermore, the frozen isentropic coefficient $\left(\gamma_{\infty}\right)$ is a decreasing function of the product $\bar{M} c_{\infty}$, see (21), which reinforces the sound acceleration in the hot zones, see (23). Thus, if the velocity of sound was only a growing function of temperature and of dissociation, the echo on the closest wall would probably be often late in regard to the other, as this one goes through the plasma core mainly when the magnetron is switched off, as illustrated in Fig. 12. However, dispersion would have a counteracting effect, if the plasma core is closer to the thermodynamic equilibrium, see (11) and Fig. 20. This would be in agreement with the temperature dependence of $\tau_{v}$, see (2). Moreover, this equation shows that $\tau_{v}$ is inversely proportional to pressure at given temperature. So it seems that when the spherical resonance is occurring, the plasma is not all the time frozen everywhere in the bulb. The value of $\tau_{v}$ in the plasma ball drops down, when the pressure is high here. As a result, the plasma ball would be going back and forth from the equilibrium state to the frozen state.

Moreover, both vibrational and chemical nonequilibrium are existing and therefore, there is a coupling between the chemical reaction and the vibrational excitation: the dissociation of a molecule is stimulated by its vibration. If somewhere in the bulb the vibrational energy is higher than its equilibrium value, the dissociation of this volume of gas is boosted as well as the light produced at its boundary by molecular recombination. Considering the strong contrast of radiance that marks the plasma ball boundary (see Fig. 1), we admit that the vibrational internal degree of freedom is frozen outside the ball and active inside, when the pressure at the center is high. Hence, assessing the maximal effect of dispersion on the average velocity of sound, the plasma is supposed to remain in the equilibrium state inside the ball and in the frozen state outside.

We derive from (10)

$$
v=v_{\infty} \sqrt{\frac{R e[\gamma]}{\gamma_{\infty}}}
$$

where $\gamma$ is given by (11). The equilibrium velocity of sound $v_{0}$ is hence

$$
v_{0}=v_{\infty} \sqrt{\frac{\gamma_{0}}{\gamma_{\infty}}}
$$

where $\gamma_{0}$ is obtained by the zero-frequency limit of (11)

$$
\gamma_{0}=\frac{2}{(1+\alpha)(2-\alpha)}\left(1+\frac{1}{\bar{M} c_{0}^{\star}}\right) .
$$

Substituting (20) into (27), as expected we obtain

$$
v_{0}=\sqrt{\gamma_{0} \frac{p}{\rho}}=\sqrt{\gamma_{0} \frac{T}{\bar{M}}} .
$$

In addition, by assessing the acoustic average of the velocity by its volume average, we are lead to

$$
\bar{v} \approx \frac{7}{8} v_{\infty}+\frac{1}{8} v_{0}
$$

as the plasma ball size is an eighth of the total bulb volume. Substituting (27) into (30), we obtain the following correction ratio

$$
\frac{\bar{v}}{v_{\infty}} \approx \frac{7+\sqrt{\gamma_{0} / \gamma_{\infty}}}{8} .
$$

This ratio is necessarily less than unity, because $\gamma_{0}<\gamma_{\infty}$. Furthermore, by considering a diatomic ideal gas $\left(\gamma_{0}=\right.$ $9 / 7$ and $\left.\gamma_{\infty}=7 / 5\right)$ a lower bound is obtained

$$
1>\frac{\bar{v}}{v_{\infty}}>0.995 .
$$

Hence, in approximating the volume average of the phase velocity of sound by its frozen value (see Fig. 11), the error is less than a percent, which is of no significant consequence in the present framework.

\section{Sound amplification due to electromagnetic coupling}

The frequency shift, that occurs when the spherical resonance appears, suggests that a spontaneous excitation is involved in the plasma ball formation. A theoretical explanation of this type of phenomenon has been given by P. M. Morse and K. U. Ingard, in the case of plane waves in a rectangular tube [44]. We adapt here their model to the case of spherical cavities.

The sound produced at the magnetron turn-off by the sudden fall in the heating of the neutral component modulates the declining electron density at the acoustic frequency [41]. The interaction between acoustic density variations and thermal energy from the electrons is taken into account, in the Appendices C and D. Indeed, as the collision frequency between electrons and molecules is an increasing function of the acoustic density, the coupling to the electromagnetic field might produce a positive feedback on the acoustic wave. Hence, the sound can be amplified by the thermal energy supplied to the neutrals by the electrons. This effect is taken into account by adding to the damped wave equation (89), see Appendix D, a source term proportional to the rate of change of the electron energy, thus proportional to the time derivative of the pressure [43]

$$
v^{2} \nabla^{2} p-\frac{1}{\tau_{a}} \frac{\partial p}{\partial t}+\frac{1}{\tau_{e}} \frac{\partial p}{\partial t}-\frac{\partial^{2} p}{\partial t^{2}}=0,
$$

where $\tau_{a}$ is the attenuation time and $\tau_{e}$ is the caracteristic time of the electromagnetic coupling, which corresponds to our measurements of the inital decay time of the afterglow (see Sect. 3.2) because the sound is mainly produced at the turn-off instants as long as the resonance is not on.

Proceeding with the same mathematical treatment as in Appendix D, we obtain from (33) the complex angular velocity $\Omega$ defined in (90), as the solution of an equation equivalent to (91) with the rate constant $\tau_{a}^{-1}-\tau_{e}^{-1}$ instead 
of $\tau_{a}^{-1}$. We transpose the results of Appendix D, finding for the imaginary part

$$
\beta=\left(\tau_{a}^{-1}-\tau_{e}^{-1}\right) / 2 .
$$

If the mode is growing, $\beta$ needs to be negative

$$
\tau_{e}<\tau_{a}
$$

which is the condition of spontaneous excitation. This means that the electrons must deliver their energy to the neutral component faster than the damping of the mode.

Regarding the actual angular velocity of the plasma response, (95) is still formally true if $\beta$ is calculated according to (34). We obtain

$$
\omega_{p}=\omega \sqrt{1-\left(\frac{\tau_{a}^{-1}-\tau_{e}^{-1}}{2 \omega}\right)^{2}} .
$$

The actual angular velocity $\omega_{p}$ is still lower than $\omega$, the resonance angular velocity if there were no damping.

For the mode to exist, the argument of the square root of (36) has to be positive. With the negative condition to the imaginary part (35), the condition of spontaneous excitation is

$$
0<\tau_{e}^{-1} / 2-\tau_{a}^{-1} / 2<\omega
$$

If $\tau_{e} \ll \tau_{a}$ in addition, we obtain

$$
1 / 2<\omega \tau_{e} \ll \omega \tau_{a},
$$

which states that an acoustic resonance can be spontaneously excited only if the electromagnetic coupling is not fast with respect to the acoustic oscillation.

In Fig. $5(\mathrm{~b})$ the measured $\omega_{c} \tau_{e} \approx 0.6>1 / 2$. Thus the plasma should be in the spherical resonance, which is not the case. Probably condition (38) is not relevant here because the condition $\tau_{e} \ll \tau_{a}$ is not applicable to the time interval shown in Fig. 5(b). As a matter of fact, the transition took place about $20 \mathrm{~ms}$ later. It might be that the resonator quality factor is very low when the plasma is not in the centralized spherical mode. In this case, it is possible that $\tau_{e} \gtrsim \tau_{a}$, so that $\beta>0$ and no spontaneous excitation occurs. Even though the vanishing of a free oscillation would require the quality factor to be very small, it might be sufficient that $\tau_{a}$ drops down temporarily to break the free oscillation.

\section{Interpretation}

\subsection{Involved normal acoustic modes}

Analytical models give the normal modes of oscillation in the case of a small amplitude in a homogeneous isothermal perfectly rigid solid sphere and they can be found in textbooks, e.g. [45]. Even though in the experiments the medium of propagation is not solid, such a model is meaningful for the resonance analysis, because the plasma is motionless at the time scale of the resonance period, as we have observed thanks to an ultrafast camera (see Sect. 3.1). Nevertheless, the medium is not homogeneous nor isothermal in the experiments, and the acoustic cavity boundary is not perfectly rigid, but the comparison with the measurements is justified when comparing relative frequencies. Used as background framework of Sect. 5, Appendix $\mathrm{C}$ recalls the basis of the standard formalism of these analytical solutions to the wave equation for spherical boundary conditions. It is now used again to determine the involved normal modes.

From the frequency $f_{\text {res }}$, at which the spherical resonance occurs, we calculate for each value of $\chi_{l, n}$ given in Table 2 (see Appendix C) the value of phase velocity the sound should have if the resonance were due to the excitation of this particular mode, so assuming $f_{\text {res }}=f_{l, n}$ in (88), see Appendix C. Even though the photo signal oscillates at a slightly lower rate than the pulse repetition frequency $f_{c}$ in the spherical resonance, since no phase locked loop was put in the control, we suppose here for simplicity that it oscillates at the frequency $f_{c}$, within an approximation error of around 1\%, see Fig. 6(c). Thereby using $f_{\text {res }} \approx f_{c}$, it follows that the sound velocity on each mode is according to (88)

$$
v_{l, n} \approx \frac{2 \pi a}{\chi_{l, n}} f_{c} .
$$

Mapping the resulting series to the volume average of the phase velocity of sound, which is approximated by the frozen velocity (error $<1 \%$, see Sect. 4.5), we finally obtain the spectrum of the possible normal modes, see Fig. 13. In the figure the horizontal axis is the spherical Bessel function order $l$ (see Appendix C) and the vertical axis is the gas temperature. For each $l$, the extremum order of the spherical Bessel function $n$, which can exist at the temperature larger than $0^{\circ} \mathrm{C}$, are shown. The measured bulb outer surface temperature of $900^{\circ} \mathrm{C}$ is shown by the dashed line. As shown by the black bars, there is only one possible pair of normal modes that can cause the beats we have observed, see Fig. $6(\mathrm{~b}):(l=0, n=2)$ and $(l=3, n=1)$. The first is indeed spherical as $l=0$, unlike the second. More details on them are given in the next sections.

\subsection{Plasma temperature}

Above all, from Fig. 13 we have obtained an important thermal assessment: the mean acoustic temperature in the bulb was about $1930{ }^{\circ} \mathrm{C}$, whereas the mean magnetron power consumption was $905 \mathrm{~W}$, corresponding to an absorbed microwave flux in the average of about $630 \mathrm{~W}$. Note that the mean acoustic temperature, i.e., the acoustic mean of the plasma temperature, is defined as the temperature of a homogeneous isothermal non deformable sphere of same radius and same resonance frequency, filled with an equivalent ideal gas, i.e. of molar mass equal to $\bar{M}$ and of isentropic coefficient equal to $\gamma_{\infty}$.

Johnston et al. have measured the temperature in the plasma core using three sulfur atomic lines in the spectrum above $800 \mathrm{~nm}$, where the spectral emission is weaker 
than in the visible part [46]. Even though they operated the magnetron in the continuous mode, the input power was in the same range as in our experiments, but with a rotating bulb. On average, they have found temperatures of $4100 \mathrm{~K} \pm 150 \mathrm{~K}$. Nevertheless, their measured value is the core temperature, whereas our value is the volumeaveraged temperature inside the whole bulb, which is given similarly with $(30)$

$$
\bar{T} \approx \frac{7}{8} T_{\text {Peri }}+\frac{1}{8} T_{\text {Core }} .
$$

Using $T_{\text {Core }}=3900^{\circ} \mathrm{C}$ and a rough estimate in the perimeter $T_{\text {Peri }}=1600^{\circ} \mathrm{C}$ results in $\bar{T} \approx 1900{ }^{\circ} \mathrm{C}$. Thus, there is no disagreement between the result of Johnston et al. and ours. With these values of the core and perimeter temperatures we get for the volume average of the sound velocity $\bar{v}_{\infty} \approx 620 \mathrm{~m} / \mathrm{s}$, i.e. less than $3 \%$ above the value obtained with our isothermal model (see Table 1). This assumption is therefore acceptable.

\subsection{Characteristics of the plasma}

The DC characteristics of the plasma at the mean acoustic temperature are given in Table 1 . The characteristic frequencies of the two involved modes are calculated with (88), see Appendix C. The spherical mode $(l=0, n=2)$ has a characteristic frequency very slightly lower than the non spherical mode $(l=3, n=1)$, respectively $28208 \mathrm{~Hz}$ and $28338 \mathrm{~Hz}$, showing a difference of $130 \mathrm{~Hz}$, i.e. only $0.5 \%$ ! A Fourier transform of the photosignal shows that the actual difference is approximately $1 \%$, see Fig. 6(c). The reason of this discrepancy of frequency shift is probably the non uniformity of the temperature in the bulb. Furthermore, this result reveals the inherent difficulty to excite only the spherical mode.

\subsection{Optimization of the luminous efficiency}

The optimization of the luminous efficiency is now regarded as a matter of nonequilibrium chemistry: the mean degree of dissociation would be optimal if the dissociationrecombination cycle was going as fast as possible, in the average throughout the bulb. This situation should be met when the peripheral layer is dominated by the recombination and the central core by the dissociation. In the frame of a one-node-lumped model, we admit that a half dissociated plasma would be optimal, i.e. if the probability of drawing from the bulb one monoatomic sulfur is twice greater than the probability of drawing one diatomic sulfur. The degree of dissociation at the mean acoustic temperature would then be $\alpha=1 / 2$ since $N_{0} \alpha=N_{0}(1-\alpha)$ according to (4).

Moreover, Fig. 10 shows that this ideal situation would be met at a mean acoustic temperature of circa $4100^{\circ} \mathrm{C}$. This is much above its actual value (see Table 1 ). It seems thus that, if the plasma could be heated up to a higher temperature, its luminous efficiency could be significantly improved. But this is limited by the mechanical resistance of the bulb at high temperature. In this regard, the formation of a stable off-wall plasma ball is certainly a key allowing to push up this limit.

\subsection{Pressure distribution}

As mentioned in the introduction, the microwave absorption is an increasing function of pressure, as observed when the sulfur evaporates during the warm-up phase. The microwave absorption is indeed due to the collisions between free electrons and molecules. This feature explains also why the plasma heating can be confined away from the bulb wall by acoustic resonance. The heat is mainly released where the pressure is a maximum at the arrival of the energy into the bulb.

Besides, mathematical modeling allows to assess the pressure distribution in each normal modes involved in the beat (see Appendix C). Using (77), (83), (84) and (86), we have graphed the nodal surfaces, see Fig. 14. It appears clearly that the non spherical mode $(l=3, n=1)$ does not fit to the plasma confinement, unlike the spherical mode $(l=0, n=2)$. In the first, indeed, the nodal surface is formed by cones with the vertex in the bulb center. So the heat release cannot be focused there, if this mode is dominating. In contrast, the nodal surface of the other mode does not pass by the bulb center, which is then the main pressure antinode, see Fig. 15. As a result, the phenomenon of sound amplification due to the electromagnetic coupling (see Sect. 5) is certainly much weaker in the non-spherical mode than in the spherical one. This would explain why stable free oscillations in the non spherical mode have not been met, see Fig. 8. And so we assume that spontaneous excitation plays a role in the resonance onset. This issue will be further developed later (see Sect. 6.8).

\subsection{Beats}

The plasma ball formation is always accompanied with a beat of the two excited normal modes. The ratio of their frequencies is according to (88) and Table 2 (Appendix C)

$$
\frac{f_{0,2}}{f_{3,1}}=\frac{\chi_{0,2}}{\chi_{3,1}}=0.9954
$$

The comparison of the measured ratio of the plasma response frequency $f_{p}$ in the spherical resonance with the pulse repetition frequency $f_{c}$ (see Fig. 16) gives a very good agreement. In the figure, the measured value is shown with the solid line and the theoretical result with the horizontal dashed line. As a result, we can state that the pulse repetition frequency is equal to the highest characteristic frequency of the two excited modes, so of mode $(l=3, n=1)$, which is therefore a forced oscillation, whereas the other $(l=0, n=2)$ is a free oscillation. And, therefore, the actual mean acoustic temperature is taken at the characteristic temperature of the mode $(l=3, n=$ 1) (see Fig. 13). 
Fig. 16 shows pointing down peaks that look like periodic stalactites, where the ratio $f_{p} / f_{c}$ decreases significantly during a few periods of the magnetron modulation, with minimum values in the range of 0.94 to 0.98 , corresponding to an amplitude below $40 \%$ of the sample maximum value (cf. Fig. 17). These stalactites occur exactly when the oscillation amplitude is shrank by the beat nodes, so when the phase shift of the microwave pulse, in respect to the plasma oscillation, lies around $225^{\circ}$ of advance shift (see Fig. 18). In these situations, the pulses of induction are recreating a new out-phased standing wave, making destructive interference. Furthermore the periodic decreases of $f_{p} / f_{c}$ is certainly due to the acoustic damping. Actually the damping slows down the oscillation, as shown mathematically in Sect. D. With (95) of Appendix $\mathrm{D}$, we have assessed the attenuation time by comparing the slow-down of the free oscillation in respect to the undamped resonance, which is given by (41),

$$
\tau_{a}^{-1}=4 \pi f_{0,2} \sqrt{1-\frac{f_{p} / f_{c}}{0.9954}} .
$$

From the minimum values reached by the ratio $f_{p} / f_{c}$ (see Fig. 16), one can estimate that $\tau_{a}$ is typically falling between $8 \mu \mathrm{s}$ and $17 \mu \mathrm{s}$ during the beat nodes. So we come to the conclusion that, for a plasma in spherical resonance, $\tau_{a}$ depends on the phase shift of the magnetron. When the microwave pulses are in a favorable phase with the free oscillation of the plasma, its forced oscillation build a constructive interference and $\tau_{a}$ reaches a maximum, around $6 \mathrm{~ms}$ according to tests of long power interruption (see Sect. 3). In contrast, when the microwave pulses are out phased, the interference is destructive and, as a result, $\tau_{a}$ passes by a minimum around $10 \mu \mathrm{s}$.

If the resonance survives the periods of outphasing as a result of spontaneous excitation, according to (35) the characteristic time of the electromagnetic coupling $\left(\tau_{e}\right)$ would have to be less than $8 \mu \mathrm{s}$. However, during the transitions to spherical resonance depicted in Fig.7 (b), $\tau_{e}$ rises above this value, so probably above the minimal value of $\tau_{a}$. Looking more carefully at this graph, nevertheless, we see that $\tau_{e}$ is in continuous decrease before the resonance onset; it rises abruptly at the very moment the photo signal modulation shrinks, which marks the resonance onset. So $\tau_{e}$ is actually smaller than $6 \mu s$ at the onset, a value below the minimum value of $\tau_{a}$, according to our preceding estimation; and thus, the spontaneous excitation might play a key role in the resonance onset.

Moreover, a closer examination of Fig. 16 shows that the frequency ratio $f_{p} / f_{c}$ rises sometimes above 0.9954 , the theoretical value it should have at undamped resonance, according to (41). In this situation the oscillation amplitude is maximum, its time derivative is consequently zero, giving momentarily a quasi steady response. Hence, $\tau_{e}$ and $\tau_{a}$ are then equal and, according to the power interruption tests reported in Sect. (3), lying in the range of milliseconds. This condition is met when the phase shift between the microwave pulse and the plasma oscillation lies around $40^{\circ}$ of advance shift (see Fig. 18). The overspeed of the oscillation in respect to the undamped reso- nance could be due to the growth of the free oscillation, which reinforces the non uniformity of the actual temperature in the bulb, increasing the modeling error by assessing $f_{p} / f_{c}$ in undamped resonance (see Sect. C). As seen in Sect. 6.5, the pressure distribution in the spherical normal mode is favorable to the sound amplification by electromagnetic coupling, which should steepen the radial profile of temperature. The phase shift giving the larger modulation amplitude corresponds to the situation of Fig. 6 , where the magnetron is switched ON when the photosignal is in the middle of its rising. We think that the oscillating pressure at the center of the bulb is then passing through its maximum, localizing here the microwave absorption. Regarding the optical radiation, we think that its maximum occurs when the core expands, since the radiation results from the molecular recombination, whose rate is strengthened by cooling. At the opposite-phase, thus when the radiation is minimum, the compression that occurs could be a strong mechanism of lag vibrational excitation as most diatomic gases have large bulk viscosities [47].

Hence we can formulate the following scenario. As seen in Fig. 7 when the standing wave builds up, $\tau_{e}$ rises sharply. Therefore $\tau_{a}$ rises also if condition (35) is true. As seen above this might happen if the phase shift of the magnetron is favorable. Then the sound amplification due to electromagnetic coupling goes on until the steady state is reached, i.e. when the variations of $\tau_{a}$ and $\tau_{e}$ cross each other in the range of 10 milliseconds. Then the outphasing reduces progressively $\tau_{a}$ which results in a shrinking of the modulation amplitude. The beat node is reached as $\tau_{a}$ and $\tau_{e}$ cross each other again, but this time at a value of about $10 \mu \mathrm{s}$, before the sound amplification restarts as (35) is fulfilled again. Hence, $\tau_{e}$ would vary cyclically at the beat frequency like $\tau_{a}$, but with some backward phase lag. The variation of $\tau_{e}$ would be due to the change in pressure distribution, which is very different in the two excited modes, as we have seen in Sect. 6.5. Indeed, $\tau_{e}$ would pass by a minimum when the spherical mode is dominant and by a maximum in the outphasing periods, when the non spherical mode is at its highest level of excitation and tends to break the plasma ball (see Fig. 14).

\subsection{Stability of the spherical resonance}

In the case depicted on Fig. 19, the plasma fell back out of resonance after a few milliseconds, like in the case of Fig. 7, having accomplished only three beat cycles. The lower graph shows the time evolution of the phase shift. As expected, this parameter is constant as long as the plasma is not in resonance and starts to change at the resonance onset. One can see, in addition, that its time derivative lies around $1 \mathrm{kHz}$. The beat is here faster than in the stable cases we met (see Fig. 6 for instance). The frequency matching seems to be not so good in this case and possibly this is the reason why the resonance did not really settle down.

In addition, as one can also see in Fig. 19(b) the phase shift starts to oscillate shortly after the timestamp $33 \mathrm{~ms}$, 
before the resonance is lost. This pattern has been observed in other experiments, and it seems therefore to be characteristic for the vanishing of the resonance. In contrast, in stable beats, like the one of Fig. 6 , the pulse repetition frequency $f_{c}$ is equal to $f_{3,1}$ (non spherical mode in forced regime) and the response frequency $f_{p}$ lies close to $f_{0,2}$ (spherical mode in free regime), see Sect. 6.6. As long as $f_{p}$ is lower than $f_{c}$, the phase shift is growing continuously, like in the beginning of the resonance time shown in Fig. 19, as indeed, the time derivative of the phase shift is by definition

$$
\dot{\phi}=2 \pi\left(f_{c}-f_{p}\right) .
$$

When the phase shift is decreasing, $f_{p}$ is greater than $f_{c}$. As the spherical mode has a high Q factor, unlike the non spherical one, see Sect. 3 , the response $\left(f_{p}\right)$ is certainly locked in the free oscillation, so more or less below $f_{0,2}$ (see Appendix D). As $f_{c}$ was constant (open loop control), the change in variation was certainly due to a rise of $f_{p}$, caused by an increase of temperature. This has probably been the result of a strong excitation of the spherical mode, since $f_{0,2}$ has exceeded $f_{c}$. As a matter of fact this new regime is not stable, the temperature decreased again and the characteristic frequencies also, as a result. Then the next matching is for the spherical mode as $f_{0,2}<f_{3,1}$, and the temperature raised again. The less excited the spherical mode is, weaker is the electromagnetic coupling (see Sect. 6.5). Hence, probably, the magnetron remained finally too long out of phase. This analysis led us to the idea that a Phase-Lock Loop control should allow to stabilize the spherical resonance on the long term.

Furthermore, vibrational relaxation might play a role in the stabilization of the plasma ball. Indeed, there might be streams of high vibrational energy everywhere throughout the bulb, as it seems to appear in the ultrafast movies, see Sect. 3.1. Thus convection might transport vibrational energy out from the ball. This is certainly a major cause of instability, because then the microwave absorption would increase in the periphery, in reason of stepwise ionization. But as seen in Sect. 4.5, the plasma ball passes into the thermodynamic equilibrium state when the wave converges back into the center, in agreement with the temperature dependence of $\tau_{v}$ as well as its pressure dependence, see (2). Thus as long as the vibrational relaxation in the ball is fast enough

$$
\forall r<a / 2, \quad \omega \tau_{v}<1,
$$

the vibrational energy exported out of the ball will be cooled down before that the next pulse of microwave comes into the bulb; then we can expect a steady energy confinement. A true confrontation of this statement to measurement is not possible here, because our examination technique is based on the analysis of the photosignal, which cannot probe into the depth of the plasma ball. Nevertheless, the light is mainly produced at the ball boundary and the measures reported on Fig. 7 show that (44) was fulfilled at the resonance onset, and it became false shortly after: the resonance did not last more than $6 \mathrm{~ms}$.
The condition (44) might not be met during the heating periods, if the magnetron is wrongly synchronized to the plasma oscillation. Indeed, as seen in Sect. 6.5, the bulb center is a pressure antinode in the spherical mode of resonance, and the lower the pressure the slower the vibrational relaxation, see (2). So if the magnetron is not in phase with the pressure at the center, the condition (44) might not be met during the heating. Again we come to the conclusion that we should be able to stabilize the ball formation thanks to a Phase-Lock Loop control.

\subsection{Spherical resonance onset}

Most of the observed plasma balls have been obtained in pulsed forced regimes with a duty-cycle in the range 20 $10 \%$. This parameter plays a key role for a given average power. The lower the duty-cycle, the more likely is the resonance onset. This can be explained by two facts:

- There is a strong shape mismatch between the bulb, which is spherical, and the microwaves, which can be regarded as plane waves.

- The acoustic growth rate, $1 /\left(2 \tau_{e}\right)$, is proportional to the electron temperature [48]. Therefore, increasing the power of the pulses is favorable to the amplification of the spherical oscillation as long as $\omega_{p} \tau_{e}>1 / 2$ according to (38). And indeed the frequency shift that appears during the resonance shows that the heavy particles become decoupled from the electrons, which means that $\tau_{e}$ increases after the transition, as our measures tend to show (see Fig. 7).

In addition, the plasma ball has been found by sweeping the repetition frequency of the microwave pulses. The criterion of spontaneous excitation (35) does not involve any frequency. In contrast, this is a determinant parameter for resonance. As seen earlier, spherical pressure distributions provide stronger electromagnetic coupling, as long as the magnetron modulation is correctly synchronized to the plasma vibration. Therefore, it seems that the spontaneous excitation is seeded by the spherical resonance, which might indeed reduce the amount of entropy contained in the bulb and, as a consequence, rises $\tau_{a}$ enough to satisfy the condition of spontaneous excitation (35).

Nevertheless, the plasma inhomogeneities could prevent the resonance, because the phase velocity of sound depends on temperature and dissociation, as seen in Sect. 4.5. Therefore, by its compensating effect, dispersion might play also a major role in the onset of the spherical resonance. Indeed, the phase velocity of sound is a growing function of $\omega \tau_{v}$ and, as one can see on Fig. 20, the dispersion is strongest at the transition from the equilibrium to the frozen state. Moreover the imaginary part of $\gamma$ is very small with respect to its real part, as shown in Fig. 21(b). Consequently, the slope of the dispersion curve is steepest at

$$
\omega \tau_{v}=c_{0}^{\star} / c_{\infty} .
$$

according to (26) and (11) [49]. The strongest compensation is expected to happen when condition (45) is met 
because the vibrational dispersion is then at its maximum. Introducing the plasma DC characteristic (Table 1) into (14), combined with (68), (73) and (74), see Appendix B, we obtain $c_{0}^{\star} / c_{\infty}=1.5$. The comparison with the measures (see Fig. 7(c)) shows good agreement.

\section{Conclusions}

Researchers at the University of Applied Sciences and Arts Western Switzerland (HES-SO) have discovered a phenomenon that allows to confine a microwave plasma in a spherical electrodeless glass bulb. If the microwave flux is pulsed in the radio frequency range $(\mathrm{kHz})$ ultrasonic waves inside the bulb are produced and, at acoustic resonance, the plasma stabilizes. In a particular resonance mode the plasma forms a centered ball of about half bulb radius. In that condition, the oscillation of pressure is largest in the centre of the bulb. Hence, if the microwave pulses are released at the moments the pressure is maximal in the centre, most of the delivered energy is absorbed there, because the microwave absorption is an increasing function of pressure. This allows to release most of the heat in the centre and the risk of melting the bulb is highly reduced. Therefore, this mode of operation permits to greatly increase the maximum power a bulb can admit ; the luminous efficiency should consequently be increased.

The present work focuses on the study of the plasma dynamics, the onset, and the stability of plasma ball formation. A fast photo sensor was used to measure the time variation of the emitted light flux. When the plasma is not in spherical resonance, the modulation of the photo signal has a regular triangular shape. On each modulation period a quasi exponential decay can be observed when the microwave power is turned off. This decay is due to the vibrational molecular relaxation. From this measurments, we can find the vibrational relaxation time, $\tau_{v}$, as well as its initial value $\tau_{e}$ to reveal the activation effect of the highly excited or dissociated molecules on vibrational relaxation.

Tests of long power interruptions have shown a characteristic time of decay of about $9 \mathrm{~ms}$, whether the plasma is in resonance or not. Comparing with [24] and with our measurements of $\tau_{v} \approx 15 \mu \mathrm{s}$, we come to the conclusion that the characteristic time of the exponential decay model increases greatly as the plasma cools down, which is in qualitative agreement with the general theory of chemical and vibrational nonequilibrium.

In spherical resonance, the photo signal modulation is almost a perfect sinusoid. In addition to this change of shape, a phase lag between the microwave modulation and the plasma oscillation appears and grows continuously: the angular frequency of the plasma oscillations $\omega_{p}$ is not anymore locked to the modulation frequency. A clear low frequency beat can be furthermore observed in the plasma response. We, therefore, made a hypothesis that in spherical resonance there are actually two normal modes simultaneously excited with a frequency difference of about $1 \%$; one has the same frequency as the microwave pulses and, thus, is in forced regime, whereas the other is in free regime.

We have calculated the phase velocity of sound as a function of temperature in order to find the series of temperatures at which a resonance would occur if the bulb were an isothermal solid sphere. There is only one pair of close characteristic frequencies in the measured temperature range, that can explain the observed beats. In addition, their frequencies agree very well with the ratio of the two beating frequencies. One of these two modes has a spherical symmetry. The comparison to the measured resonance frequency gives a mean acoustic temperature of $1930{ }^{\circ} \mathrm{C}$, whereas the mean magnetron power consumption was $905 \mathrm{~W}$ and the maximum of the bulb surface temperature $900{ }^{\circ} \mathrm{C}$. Furthermore, by estimating that half-dissociation would maximize the rate of the dissociation/recombination cycles throughout the bulb, we have been lead to speculate that, if the plasma would be heated up to a higher temperature, the luminous efficiency could reach much higher values. However, the mechanical resistance of the bulb sets a high temperature limit for its operation. In this regard, the stable formation of a plasma ball at distance from the wall is certainly a key to push up this operation limit.

For the development of an automatic control system, we have studied the transition into the spherical resonance. The transition is marked by a sudden shrinking of the modulation amplitude of the photo signal. At this very moment, a sudden rise of $\tau_{e}$ can be observed, as the product $\omega_{p} \tau_{e}$ sharply increases from its initial value of about 1 to a value larger than 5 . In addition, the frequency shift that appears at the resonance shows that the heavy particles become decoupled from the electrons which means, too, that $\tau_{e}$ increases. This observation is theoretically confirmed, since we have shown mathematically that an acoustic resonance can be spontaneously excited by sound amplification, due to electromagnetic coupling, only if the coupling is slow with respect to the acoustic oscillation.

We have shown, also theoretically, that any normal mode of a spherical cavity can be spontaneously excited by electromagnetic coupling, if the attenuation time $\tau_{a}$ is greater than $\tau_{e}$. It was estimated through tests of long power interruption that $\omega_{p} \tau_{a}$ is in the range of $10^{3}$ at the beat antinodes and around 2 at the beat nodes. It seems, therefore, that the condition mentioned above, $\tau_{a}>\tau_{e}$, was fulfilled in the spherical resonance. Thus, we can postulate the following scenario. The resonant free oscillation is onset due to the spontaneous excitation. Its mode is favorable to sound amplification by electromagnetic coupling, because the pressure antinode is at the bulb center. In contrast, the forced oscillation is a non spherical mode, which has a pressure node at the center. At the beat antinode the variations of $\tau_{a}$ and $\tau_{e}$ cross each other in the range of $6 \mathrm{~ms}$. Then the outphasing reduces progressively $\tau_{a}$ resulting in a compression of the modulation amplitude. The beat node is reached when $\tau_{a}$ and $\tau_{e}$ cross each other again, this time around a value of $10 \mu \mathrm{s}$, before the sound amplification restarts, since the condition $\tau_{a}>\tau_{e}$ is fulfilled again. 
Furthermore, the acoustic growth rate due to the electromagnetic coupling, $\tau_{e}^{-1} / 2$, is proportional to the energy of free electrons. Thus, increasing the power of the pulses is favorable to spontaneous excitation, as long as $\tau_{e}^{-1} / 2<\omega+\tau_{a}^{-1} / 2$, because otherwise the mode would not exist. Indeed, the duty-cycle plays actually a role. For a given average power, the lower was the duty-cycle, the more likely was the resonance onset.

The strong contrast in the light emission on the plasma ball boundary is certainly due to the interplay between the chemical reactions and the vibrational relaxation. We can consider the plasma ball formation as the separation of two gases. Outside the boundary is a gas of frozen vibrational internal degree of freedom, while inside the boundary a weakly ionized plasma is oscillating between the equilibrium state and the frozen state. Through the resulting 'pumping' effect, occurring inside the plasma ball, the vibrational degree of freedom reaches there high levels of activation. Simultaneously, the stepwise ionization enhances the molecular dissociation, if the magnetron modulation is in phase with the pressure oscillation. The strong contrast in light emission can then be understood as the effect of diffusion of monoatomic sulfur at the ball boundary, as the photons are generated by the electronic relaxation of recombined diatomic sulfur. Therefore, dispersion might play also an important role in the onset of the spherical resonance. On one hand, the sound propagation is slower when the gas is in the equilibrium state than when it is in the frozen state. But on the other hand, the rise of temperature and dissociation during the heating periods tends to accelerate the propagation through the plasma ball. Thereby, dispersion may compensate this effect. The strongest compensation is expected at $\omega \tau_{v}=c_{0}^{\star} / c_{\infty}$. In our experiments, see Table $1, c_{0}^{\star} / c_{\infty}=1.5$, which is in agreement with the measurements performed at the transition to the spherical resonance.

We think that the vibrational relaxation also plays a role in the stabilization of the plasma ball formation. Indeed, convectional transport of vibrational energy out from the plasma is certainly a major cause of instability, because the microwave absorption would then increase in the periphery. The plasma is thought to settle in the thermodynamic equilibrium, when the central pressure is high. Thus, as long as the vibrational relaxation in the ball is fast enough the vibrational energy exported out of the ball is cooled down before the next pulse of microwave and we can expect a steady energy confinement. However, this condition might not be fulfilled anymore when the magnetron is badly synchronized with the plasma oscillation. Hence, we arrive to conclude that the ball formation could be stabilized on the long term by the use of a Phase-Lock Loop control.

Finally, we want to emphasize that this work is much related to interpretations. There is still a lot of work to be done. Modeling the excited state kinetics is necessary to describe fully the complex phenomena that occur in the bulb. Such a task is out of scope in the present study, but we hope that it will be achieved in the future, as nowadays the distribution functions of the electron energy, as well as the vibration and electronic states, can be calculated by numerical simulation taking into account optical thickness and plasma geometry [20]. Deeper understanding of the interplay between the plasma chemistry and the acoustics, as required in our analysis of the plasma ball formation, might lead to futher discoveries in plasma sonochemistry.

\section{Acknowledgement}

The authors would like to thank the Swiss Foundation 'Gerbert Rüf Stiftung' for a support of our work with funding (contract GRS-023/14). Our thank goes also to the company Lumartix, who has provided specific components of the prototype, like the light bulbs and the microwave applicator. The authors are also grateful to Dr. Seth Putterman, expert in sonoluminescence and dense plasma at the University of California, Los Angeles (UCLA), who made important scientific contributions to this work by intense and helpful discussions with Dr. Gilles Courret, mainly in sabbatical visit to UCLA.

\section{Author contribution statement}

The plasma ball formation reported in the present paper has been discovered by O. Gudozhnik and G. Courret, who was the project leader. G. Courret developed the modeling (see Sect. 4) including the four Appendices (see Sect. A to D), with the advices of M. Croci and P. W. Egolf. G. Courret and P. Nikkola wrote the manuscript, with the revisions of J. Braun, M. Croci and P. W. Egolf. S. Wasterlain was in charge of the setup, especially the data acquisition system; he developed the PC interface. O. Gudozhnik developed the high frequency pulse generator and its PC interface. S. Gavin developed the pulsed high voltage unit for the magnetron. The measurements were performed by G. Courret, S. Wasterlain and O. Gudozhnik. G. Courret and P. Nikkola applied the acoustic resonance model and found the onset conditions of the spherical resonance. P. Nikkola developed a method to get the phase-shift from the measured signal. M. Girardin has supported engineering aspects.

\section{A Thermodynamic model}

The gas in the bulb is assumed to be a chemically reacting mixture of ideal gases. Therefore, in contrast to the mass, the number of molecules in the bulb is not constant and, according to the first principle of thermodynamics, it follows that [50]

$$
c_{p}-c_{v}=\left.\left(\left.\frac{\partial e}{\partial \rho^{-1}}\right|_{T}+p\right) \cdot \frac{\partial \rho^{-1}}{\partial T}\right|_{p},
$$

where $e$ is the internal energy per unit of mass. Using in addition the ideal gas law leads to

$$
\frac{c_{p}}{c_{v}}=1+\frac{1}{\bar{M} c_{v}}\left(\left.\frac{1}{p} \frac{\partial e}{\partial \rho^{-1}}\right|_{T}+1\right) .
$$


We model the internal energy of sulfur as a threelevels system (see Fig. 22). Sulfur molecules are sorted in three categories: diatoms in the ground electronic state $X^{3} \sum_{g}^{-}$, diatoms in the excited electronic state $B^{3} \sum_{u}^{-}$, and monoatoms, whose respective numbers are $N_{2}^{\circ}, N_{2}^{\star}$, and $N_{1}$.

Adopting the rigid-rotator model for the rotational energy of the diatomic molecules [51], the internal energy of the gas is (see Fig. 22):

$$
\begin{gathered}
\mathcal{E}=\left(5 N_{2}+3\left(N_{1}+N_{A}\right)\right) T / 2+ \\
N_{2}^{\star}\left(E_{v b}+h \nu_{m}\right)+N_{2}^{\circ} E_{v b}+N_{1} D / 2,
\end{gathered}
$$

where $E_{v b}$ is the average vibrational energy of the diatoms that are in the ground electronic state and $h \nu_{m}$ the average of the emitted photon energy (see Fig. 22), where $h$ is the Planck constant and $\nu_{m}$ the corresponding frequency.

Let us consider now the term of (48) that does not explicitly depend on temperature:

$$
\mathcal{E}_{S}^{e l}=\xi N_{0} h \nu_{m}+N_{2} E_{v b}+N_{1} D / 2
$$

where $\xi=N_{2}^{\star} / N_{0}$ is the degree of excitation. Besides, measuring the decay rate of the fluorescence at $370 \mathrm{~nm}$, McGee et al. have determined the lifetime of the excited state $B^{3} \sum_{u}^{-}$to be $\tau_{B}=45 \mathrm{~ns}$ [53], whereas in [54] a value $20 \mathrm{~ns}$ was given. Hence, we can assess the number of excited diatoms from the emitted optical flux $\Phi_{\text {opt }}$ by the following approximation [55]

$$
N_{2}^{\star} \approx \frac{\Phi_{o p t} \tau_{B}}{h \nu_{m}} .
$$

Our measurements give $\Phi_{\text {opt }} \tau_{B} \approx 5 \mu \mathrm{J}$. Besides, the integration of the emission spectrum, when the acoustic resonance is onset (see Fig. 23), gives $h \nu_{m}=2.19 \mathrm{eV}$. This value is however slightly higher, at $2.25 \mathrm{eV}$, in absence of resonance. Such spectral shifts were already observed in the case of non spherical resonances [56]. This might result from the acoustic absorption due to the vibrational degree of freedom. Indeed, the more vibrations in a diatom, the larger its mean internuclear distance. The energy diagram of disulfur shows that the higher the vibrational excitation level, the closer to the ground state the excited electronic state is [57]. And so, because of the Franck-Condon principle, the higher the vibrational excitation, the lower the energy of the emitted photon is. As a result, the vibrational excitation of disulfur yields a red shift of the maximum of the spectrum, like in the effect of pressure rise [58].

As one can see, furthermore on Fig. 23, the profile of the emission spectrum does not fit to the Planck's law, whatever the black-body temperature is. This may appear inconsistent with the conclusion of Sect. 3.1, in regard to the optical thickness of the plasma. But in fact, there is at least one cause for such a mismatch with the black-body radiation: strong absorption occurs for resonance lines, which are due to transitions between the fundamental electronic level and the first excited electronic state [59]. The radiation of disulfur in the optical spectral range is composed by a series of resonance lines, so close to each other, that they form a continuous spectrum, as in addition, the high-pressure operating condition broadens the lines [60].

So substituting $h \nu_{m}=2.19 \mathrm{eV}$ into (50), we have assessed the mean density of excited diatoms, $N_{2}^{\star} / V$, which is about $10^{12} \mathrm{~cm}^{-3}$, being seven orders of magnitude below $n_{0}$, the molar density of sulfur if there were no dissociation $\left(n_{0}=N_{0} / V: 1.7 \times 10^{19} \mathrm{~cm}^{-3}\right.$ in the present case). The value $\xi<10^{-7}$ results and, consequently, $\xi h \nu_{m}$ is negligible with respect to $E_{v b}$. Hence, using also (4), we simplify (49) as follows

$$
\mathcal{E}_{S}^{e l} / N_{0}=(1-\alpha) E_{v b}+\alpha D
$$

As the mass of gas is given by $\rho V$, the internal energy per unit mass is obtained by dividing (48) by this quantity

$$
e=\frac{\left(5 N_{2}+3\left(N_{1}+N_{A}\right)\right) T / 2+\mathcal{E}_{S}^{e l}}{\rho V} .
$$

Since argon is only present as a trace

$$
N_{A} \ll N_{0}
$$

we get, making use of (4),

$$
\frac{\rho}{n_{0}} e=(\alpha+5) T / 2+(1-\alpha) E_{v b}+\alpha D .
$$

The partial derivative of the internal energy per unit of mass can be decomposed as follows

$$
\left.\frac{\partial e}{\partial \rho^{-1}}\right|_{T}=\left.\left.\frac{\partial \alpha}{\partial \rho^{-1}}\right|_{T} \frac{\partial e}{\partial \alpha}\right|_{T} .
$$

Using (54), we have

$$
\left.\frac{\partial e}{\partial \alpha}\right|_{T}=\frac{n_{0}}{\rho}\left(\frac{T}{2}+D-E_{v b}\right) .
$$

Regarding the first term in the product on the right hand side of (55), we derive the ideal gas law at a constant temperature with respect to the degree of dissociation

$$
\left.\bar{M} p \frac{\partial \rho^{-1}}{\partial \alpha}\right|_{T}=-\left.\bar{M} \rho^{-1} \frac{\partial p}{\partial \alpha}\right|_{T}-\left.p \rho^{-1} \frac{\partial \bar{M}}{\partial \alpha}\right|_{T} .
$$

Besides, for a total pressure $p, \alpha$ is given by [61]

$$
\alpha=\sqrt{\frac{K_{e}(T)}{4 p+K_{e}(T)}},
$$

where

$$
K_{e}(T)=\frac{p_{1}^{2}}{p_{2}}
$$

is the equilibrium constant, which depends on temperature, but not on pressure. Since it is constant at constant temperature, the inverse of the first partial derivative on the right hand side of (57) is

$$
\left.\frac{\partial \alpha}{\partial p}\right|_{T}=\frac{-2 \alpha}{4 p+K_{e}(T)} .
$$


Moreover, the pressure is high, so $p \gg K_{e}(T)$ in the major part of the bulb (see Table 1) and, consequently,

$$
\left.\frac{\partial p}{\partial \alpha}\right|_{T} \approx \frac{-2 p}{\alpha}
$$

The second derivative on the right hand side of (57) is obtained from (5)

$$
\left.\frac{\partial \bar{M}}{\partial \alpha}\right|_{T}=\frac{-N_{0} \bar{M}}{N_{A}+N_{0}(1+\alpha)} .
$$

We finally obtain for (57)

$$
\left.\frac{\partial \rho^{-1}}{\partial \alpha}\right|_{T}=\rho^{-1}\left(\frac{2}{\alpha}+\frac{N_{0}}{N_{A}+N_{0}(1+\alpha)}\right) .
$$

Applying (53), we arrive at

$$
\left.\frac{\partial \alpha}{\partial \rho^{-1}}\right|_{T} \approx \rho \frac{\alpha(1+\alpha)}{2+3 \alpha} .
$$

Substituting (64) and (56) into (47) yields

$$
\frac{c_{p}}{c_{v}} \approx 1+\frac{1}{\bar{M} c_{v}}\left(1+\frac{n_{0} \alpha(1+\alpha)}{p(2+3 \alpha)}\left(\frac{T}{2}+D^{\star}\right)\right),
$$

where the effective dissociation energy is

$$
D^{\star}=D-E_{v b} .
$$

Neglecting the partial pressure of argon, the ideal gas equation gives $p \approx n_{0}(1+\alpha) T$ and (65) simplifies to

$$
\frac{c_{p}}{c_{v}} \approx 1+\frac{1}{\bar{M} c_{v}}\left(1+\frac{\alpha}{2+3 \alpha}\left(\frac{1}{2}-\frac{D^{\star}}{T}\right)\right) .
$$

\section{B Dispersion due to vibrational relaxation}

The gas is dispersive, as discussed in Sect. 4.2: the phase velocity of sound is a function of the relaxation time $\tau_{v}$ through the dependence of the isentropic coefficient to this parameter, see (11). Indeed, the heat capacity at thermodynamic equilibrium is the sum of the reactive and the frozen ones,

$$
c_{0}=c_{r}+c_{\infty}
$$

where $c_{\infty}$ is given by (24). On the other hand, the reactive heat capacity, $c_{r}$, is due to the dissociation reaction of sulfur $[42]$

$$
c_{r}=\left.\left.\frac{\partial e}{\partial \alpha}\right|_{V, T} \frac{\partial \alpha}{\partial T}\right|_{V} .
$$

To calculate this component, we first make use of (56)

$$
c_{r}=\left.\frac{n_{0}}{\rho}\left(\frac{T}{2}+D^{\star}\right) \frac{\partial \alpha}{\partial T}\right|_{V} .
$$

Derivation of (19) leads to

$$
\left.\frac{\partial \alpha}{\partial T}\right|_{V}=\frac{\alpha^{3}}{2-\alpha} \frac{4 n_{0}}{K_{e}(T)}\left(1-\frac{T K_{e}^{\prime}(T)}{K_{e}(T)}\right),
$$

and derivation of (16) results in the ratio

$$
\frac{K_{e}^{\prime}(T)}{K_{e}(T)}=\frac{1}{T}\left(\frac{1}{2}+\frac{D}{T}\right) .
$$

Applying (23) and $(1+\alpha) \bar{M} \approx \rho / n_{0}$, we arrive at

$$
\frac{c_{r}}{c_{\infty}} \approx \frac{2 n_{0}\left(2 D^{\star}+T\right)(2 D-T) \alpha^{3}}{T K_{e}(T)(2-\alpha)\left(7-\alpha+3 N_{A} / N_{0}\right)} .
$$

Combining this result with (24) allows to calculate $c_{r}$, as well as $c_{0}$ from (68).

In order to determine the complex isentropic coefficient, see (11), we still need to calculate the effective isochoric equilibrium heat capacity per unit of mass $c_{0}^{\star}$, see (14), as well as the effective dissociation energy $D^{\star}$, see (66). This second parameter is known at thermodynamic equilibrium as the vibrational energy is then a function of temperature determined by quantum mechanics. In this regard, we use the fundamental vibrational frequency of disulfur, which has been measured as the ground state energy of these molecules. Spectroscopy has provided $\nu_{0}=$ $727.848 \mathrm{~cm}^{-1}$ [57], in consistance with the zero-point energy of vibrations given in [32] $(0.089 \mathrm{eV}$, see Fig. 22). This corresponds to a characteristic temperature of $T_{0}=$ $h \nu_{0}=1047 \mathrm{~K}$, such that the vibrational energy at thermodynamic equilibrium is [62]

$$
E_{v b 0}=\frac{T_{0}}{e^{T_{0} / T}-1} .
$$

Now, we have all the terms in (28) to calculate the phase velocity of sound (27) at the equilibrium limit from (28) and (29), as shown in Fig. 11, as well as at any frequency from (10) and (11), as shown in Fig. 20.

\section{Acoustic resonance in a solid isothermal sphere}

We start from the standard theory of acoustic resonance in an isothermal solid sphere in order to identify all the normal modes as well as a geometrical equation that they must all fulfill (78). Doing so, we have been able to remove the spatial coordinates from the damped wave equation (91).

The acoustic wave equation for small variations of pressure $p$ is $[63]$

$$
\nabla^{2} p-\frac{1}{v^{2}} \frac{\partial^{2} p}{\partial t^{2}}=0
$$

where $v$ is the phase velocity of sound. In the spherical coordinates with $r$ (radius), $\theta$ (zenith angle), and $\phi$ (azimuthal angle), the Laplacian of pressure is

$$
\begin{aligned}
& \nabla^{2} p=\frac{1}{r^{2}}\left[\frac{\partial}{\partial r}\left(r^{2} \frac{\partial p}{\partial r}\right)+\right. \\
& \left.\frac{1}{\sin \theta} \frac{\partial}{\partial \theta}\left(\sin \theta \frac{\partial p}{\partial \theta}\right)+\frac{1}{\sin ^{2} \theta} \frac{\partial^{2} p}{\partial^{2} \phi}\right]
\end{aligned}
$$


In the case of small perturbations $v$ is independent of the wave amplitude, and we can describe the pressure as a composition of oscillatory functions of angular frequency $\omega$ and use the method of separation of variables

$$
p=A R(x) \Theta(\theta) \Phi(\phi) e^{i \omega t}
$$

where $x$ is the non-dimensional radius $x=\omega r / v$. Introducing (77) into (75) results in

$$
g(R(x), \Theta(\theta), \Phi(\phi))=-x^{2},
$$

where

$$
\begin{aligned}
& g(R(x), \Theta(\theta), \Phi(\phi))=\frac{1}{R(x)} \frac{d}{d x}\left(x^{2} R^{\prime}(x)\right)+ \\
& \frac{1}{\Theta(\theta)} \frac{1}{\sin \theta} \frac{d}{d \theta}\left(\sin \theta \Theta^{\prime}(\theta)\right)+\frac{1}{\Phi(\phi)} \frac{\Phi^{\prime \prime}(\phi)}{\sin ^{2} \theta} .
\end{aligned}
$$

Introducing (79) and (78) into (76) leads to

$$
\nabla^{2} p=-p\left(\frac{\omega}{v}\right)^{2}
$$

For the modes of spherical symmetry, or shortly spherical modes, $g$ reduces to

$$
g=g(R(x))=\frac{1}{R(x)}\left(x^{2} R^{\prime \prime}(x)+2 x R^{\prime}(x)\right) .
$$

Substituting (81) to (78) we get for the spherical modes the solution $R(x)=\sin (x) / x$.

Furthermore we impose the boundary condition of an nondeformable sphere of radius $a$

$$
\left.\frac{\partial p}{\partial x}\right|_{x=\omega a / v}=0 .
$$

The solutions of (78) which satisfy (82) has a sinusoidal dependence in the azimuthal angle [45]

$$
\Phi(\phi)=\cos \left(m \phi+\gamma_{m}\right),
$$

where $m$ is an integer constant of separation of variables and $\gamma_{m}$ is a constant angle that depends on the initial conditions.

The dependence on the zenith angle is an associated Legendre polynomial of the first kind of order $l$ and degree $m$ where $l(l+1)$ is the square of the second constant of separation of variables and it must satisfy $m \leq l$ [45]

$$
\Theta(\theta)=\mathcal{P}_{l}^{m}(\cos (\theta)),
$$

with $\mathcal{P}_{l}^{m}$ being the Legendre polynomial of order $l$ and degree $m$. Finally, the radial dependence is the solution of the radial Sturm-Liouville differential equation

$$
x^{2} R^{\prime \prime}(x)+2 x R^{\prime}(x)+\left(x^{2}-l(l+1)\right) R(x)=0 .
$$

For any spherical mode $(85)$ reduces to $l(l+1) R(x)=0$ as $(78)$ reduces to $x^{2} R^{\prime \prime}(x)+2 x R^{\prime}(x)=-x^{2} R(x)$ and therefore $l$ must be zero. In the opposite case, (78) implies, moreover, that the standing wave is not of spherical symmetry.

As the pressure is finite in the origin, the radial dependence can only be a spherical Bessel function of the first kind and of order $l$

$$
R(x)=j_{l}(x)
$$

The boundary condition (82) is thus an extremum of $(86)$

$$
\frac{\omega}{v} a=\chi_{l, n},
$$

where $n$ is the order of the extremum. The constants $\chi_{l, n}$ are given in table 2 . All the normal modes of a single pair $(l, n)$ have the same natural frequency, which from (87), is

$$
f_{l, n}=\frac{v \chi_{l, n}}{2 \pi a} .
$$

This equation determines the series of the characteristic frequencies, once the sound phase velocity is known. We have applied this in Sect. 6 to find the possible modes that can be co-excited, in order to explain the beats that we have observed.

\section{Damped acoustic oscillations}

This generalization has lead us to a necessary condition to the sound amplification by electromagnetic coupling, whatever the excited mode (37). To add damping of the acoustic oscillations, the wave equation (75) becomes

$$
\nabla^{2} p-\frac{1}{v^{2} \tau_{a}} \frac{\partial p}{\partial t}-\frac{1}{v^{2}} \frac{\partial^{2} p}{\partial t^{2}}=0
$$

where $\tau_{a}$ is the attenuation time. Admitting that the acoustic loss rate is proportional to the wave energy $W$, the attenuation time is $\tau_{a}=-2 W / \dot{W}$. Introducing into (89) the generalized form of the pressure $p=A R(x) \Theta(\theta) \Phi(\phi) e^{i \Omega t}$ where $\Omega$ is now a complex angular velocity,

$$
\Omega=\omega_{p}+i \beta
$$

with $\beta$ being the damping rate and, as before, $x=\omega r / v$ and $(R(x), \Theta(\theta), \Phi(\phi))$ being a real solution of (78) so that $(80)$ is applicable, we obtain

$$
\Omega^{2}-\frac{i}{\tau_{a}} \Omega=\omega^{2} .
$$

According to this equation, the complex angular velocity $\Omega$ has a non-zero real part only if

$$
\tau_{a} \omega>1 / 2
$$

Omitting the negative-signed solution since a negative real frequency is not physical, the equation (91) gives

$$
\Omega=\frac{\sqrt{\left(2 \tau_{a} \omega\right)^{2}-1}+i}{2 \tau_{a}} .
$$


We note that the module of $\Omega$ is equal to $\omega$ and that the sinus of its phase is $1 /\left(2 \tau_{a} \omega\right)$. Moreover, its imaginary part

$$
\beta=1 /\left(2 \tau_{a}\right)
$$

is positive; the oscillation is indeed damped in time at a rate of $\exp \left(-t /\left(2 \tau_{a}\right)\right)$. Furthermore, its real part gives the actual angular velocity of the response

$$
\omega_{p}=\omega \sqrt{1-\left(\frac{\beta}{\omega}\right)^{2}},
$$

which is lower than $\omega$, which denotes the resonance angular velocity in the absence of damping.

\section{References}

1. M. Capitelli et al:, Fundamental aspects of plasma chemical physics, vol. Kinetics, (Springer, 2016) p. 228

2. A. Fridman and L. A. Kennedy, Plasma Physics and Engineering (CRC Press, USA 2011) 28-31, p. 185 - 186

3. C. W. Johnston, Ph.D. thesis, Technische Universiteit Eindhoven, 2003, p.14

4. C. W. Johnston, Ph.D. thesis, Technische Universiteit Eindhoven, 2003, p.15

5. H. W. P. van der Heijden and J.J.A.M. van der Mullen, J. Phys. B: At. Mol. Opt. Phys. 34, (2001) p. 4183 - 4201

6. M. G. Ury, C. H. Wood, and J. T. Dolan, Lamp including sulfur, USA Patent no. 5,404,076, 1995

7. G. Courret, L. Calame, M. Croci, P. Egolf and A. Meyer, in Proceedings of the 14 Schweizerisches Status-Seminar Energie- und Umweltforschung im Bauwesen (Zurich, Switzerland, 2006) p. 5

8. A. Meyer, G. Courret and M. Croci, Plasma lamp with means to generate in its bulb a resonant ultrasound wave, Patent pending EP 1876633 A1

9. G. Courret, L. Calame, J. Croisier, M. Croci and A. Meyer, in Proceedings of the 15. Schweizerisches StatusSeminar Energie- und Umweltforschung im Bauwesen (Zurich, Switzerland, 2008) p. 4

10. G. Courret, L. Calame, J. Croisier, M. Croci and A. Meyer, in Proceedings of the 15. Schweizerisches StatusSeminar Energie- und Umweltforschung im Bauwesen (Zurich, Switzerland, 2008) p. 7

11. S. Gavin, M. Carpita and G. Courret, Proceedings of the 16th Conference on Power Electronics and Applications (EPE14-ECCE Europe, Lappenranta, Finland, 2014)

12. C. W. Johnston, H. W. P. van der Heijden, A. Hartgers, K. Garloff, J. van Dijk and J. J. A. M. van der Mullen, J. Phys. D: Appl. Phys. 37, (2004) p. 211 - 220

13. G. Courret, L. Calame, J. Croisier, M. Croci and A. Meyer, in Proceedings of the 15. Schweizerisches StatusSeminar Energie- und Umweltforschung im Bauwesen (Zurich, Switzerland, 2008) p. 3 - 4

14. A. Fridman and L. A. Kennedy, Plasma Physics and Engineering (CRC Press, USA 2011) p. 97 - 99

15. A. Fridman and L. A. Kennedy, Plasma Physics and Engineering (CRC Press, USA 2011) p. 100 - 101

16. A. Fridman and L. A. Kennedy, Plasma Physics and Engineering (CRC Press, USA 2011) p. 257 - 261
17. G. Colonna, V. Laporta, R. Celiberto, M. Capitelli and J. Tennyson, Plasma Sources Sci. Technol. 24, (2015) 035004 (9pp)

18. P. M. Morse and K. U. Ingard, Theoretical Acoustics (Princeton University Press, USA 1968) p. 785

19. L. D. Pietanza, G. Colonna, G. D'Ammando and M. Capitelli, Plasma Phys. and Control. Fusion. 59, (2017) 014035 (8pp)

20. M. Capitelli, G. Colonna, G. D'Ammando, K. Hassouni, A. Laricchiuta and L. D. Pietanza, Plasma Process. and Polym. 14, (2017) 1600109

21. A. Fridman and L. A. Kennedy, Plasma Physics and Engineering (CRC Press, USA 2011) p. 116 - 120

22. C. D'Ammando, G. Colonna, M. Capitelli and A. Laricchiuta, Phys. of Plasmas 22, (2015) 034501

23. G. Colonna, C. D'Ammando and L. D. Pietanza, Plasma Sources Sci. Technol. 25, (2016) 054001 (12pp)

24. C. W. Johnston and J.J.A.M. van der Mullen, J. Phys. D: Appl. Phys. 37, (2004) p. 573 - 587

25. M. Capitelli, G. Colonna, C. D'Ammando, V. Laporta and A. Laricchiuta, Chemical Physics 438, (2014) 31-36

26. J. D. Anderson Jr. Hypersonic and High-Temperature Gas Dynamics (AIAA Education series, Second edition, USA 2006) p. 583

27. J. D. Anderson Jr. Hypersonic and High-Temperature Gas Dynamics (AIAA Education series, Second edition, USA 2006) p. 584

28. A. Fridman and L. A. Kennedy, Plasma Physics and Engineering (CRC Press, USA 2011) p. 118

29. C. Park, Nonequilibrium Hypersonic Aerothermodynamics (A Wiley-Interscience publication, USA 1990) p. 58

30. P. M. Morse and K. U. Ingard, Theoretical Acoustics (Princeton University Press, USA 1968) p. 781

31. H. Rau, T. R. N. Kutty, and J. R. F. Guedes de Carvalho, J. Chemical Thermodynamics 5, (1973) p. $291-302$

32. B. Meyer, Chemical Reviews 76(3), (1976) p. $367-388$

33. M. Capitelli, G. Colonna, and A. D'Angola, Fundamental Aspects of Plasma Chemical Physics, vol. Thermodynamics (Springer, 2012) p. 26

34. M. Capitelli, G. Colonna, and A. D'Angola, Fundamental Aspects of Plasma Chemical Physics, vol. Thermodynamics (Springer, 2012) p. 28

35. W. Massey, Electronic and ionc impact phenomena, vol. III Slow Collisions of Heavy Particles (Oxford University Press, UK 1971) p. 1467

36. M. Capitelli, G. Colonna, and A. D'Angola, Fundamental Aspects of Plasma Chemical Physics, vol. Thermodynamics (Springer, 2012) p. 8

37. M. A. Reno, B. J. McBride and S. Gordon, Coefficients for calculating thermodynamic and transport properties of individual species, Technical report, NASA, 1993

38. L. Landau and E. Lifchitz, Physique statistique (Editions Mir, Russia 1994) p. 337

39. C. W. Johnston, Ph.D. thesis, Technische Universiteit Eindhoven, 2003, p. 22

40. G. Courret, L. Calame, M. Croci, P. Egolf and A. Meyer, in Proceedings of the 14 Schweizerisches Status-Seminar Energie- und Umweltforschung im Bauwesen (Zurich, Switzerland, 2006) p. 7

41. P. M. Morse and K. U. Ingard, Theoretical Acoustics (Princeton University Press, USA 1968) p. 786

42. M. Capitelli, G. Colonna, and A. D'Angola, Fundamental Aspects of Plasma Chemical Physics, vol. Thermodynamics (Springer, 2012) p. $22-25$ 
43. P. M. Morse and K. U. Ingard, Theoretical Acoustics (Princeton University Press, USA 1968) p. 787

44. P. M. Morse and K. U. Ingard, Theoretical Acoustics (Princeton University Press, USA 1968) p. 790 - 791

45. L. E. Kinsler, A. R. Frey, A. B. Coppens and J. V. Sanders, Fondamental of acoustics (Fourth edition, John Wiley \& Sons, Inc, USA 2000) p. 250 - 252

46. C. W. Johnston, J. Jonkers and J. J. A. M. van der Mullen,

J. Phys. D: Appl. Phys. 35, (2002) p. 2578 - 2585

47. M. S. Cramer, Physics Of Fluids, 24, (2012) 066102

48. P. M. Morse and K. U. Ingard, Theoretical Acoustics (Princeton University Press, USA 1968) p. 788

49. W. Massey, Electronic and ionc impact phenomena, vol. III Slow Collisions of Heavy Particles (Oxford University Press, UK 1971) p. 1468

50. J. D. Anderson Jr. Hypersonic and High-Temperature Gas Dynamics (AIAA Education series, Second edition, USA 2006) p. 480

51. C. Park, Nonequilibrium Hypersonic Aerothermodynamics (A Wiley-Interscience publication, USA 1990) p. 18 - 25

52. E.G. Leksina, E.A. Mukhina, Yu.V. Pavlov, R.M. Umarkhodzhaev, O.M. Vokhnik and A.N. Kozlov, Physics of Vibrations 8(3), (2000) p. 158 - 164

53. T. H. McGee and R. E. Weston, J. Chem. Phys. Lett. 47(352), (1977) p. 352

54. A. Fridman and L. A. Kennedy, Plasma Physics and Engineering (CRC Press, USA 2011) p. 77

55. A. Fridman and L. A. Kennedy, Plasma Physics and Engineering (CRC Press, USA 2011) p. 424

56. G. Courret, L. Calame, M. Croci, P. Egolf and A. Meyer, in Proceedings of the 14 Schweizerisches Status-Seminar Energie- und Umweltforschung im Bauwesen (Zurich, Switzerland, 2006) p. 6

57. D. A. Peterson and L. A. Schlie, J. Chem. Phys. 73(4), (1980) p. $1551-1566$

58. H. van der Heijden, J. van der Mullen, J. Baier and A. Krber, J. Phys. B: At. Mol. Opt. Phys. 35 (2002) p. 3633 3654

59. M. I. Boulos, P. Fauchais and E. Pfender, Thermal Plasma, vol. I Fundamentals and Applications (Springer, 1994) p. 377

60. A. Fridman and L. A. Kennedy, Plasma Physics and Engineering (CRC Press, USA 2011) p. 427 - 429

61. M. Capitelli, G. Colonna, and A. D'Angola, Fundamental Aspects of Plasma Chemical Physics, vol. Thermodynamics (Springer, 2012) p. 6

62. C. Park, Nonequilibrium Hypersonic Aerothermodynamics (A Wiley-Interscience publication, USA 1990) p. 14

63. L. E. Kinsler, A. R. Frey, A. B. Coppens and J. V. Sanders, Fundamentals of acoustics (Fourth edition, John Wiley \& Sons, Inc, USA 2000) p. 119 


\section{List of Figures}

1 Left: The plasma in spherical resonance has a spherical shape and, despite gravitation, it is confined in the middle of the bulb. Right: Plasma out of resonance has an irregular shape accompanied with random ejections of plasma. . . . . . . . . . . . . . . . . . . . . . .

2 Schematic of the experiment including the control PC, the pulse generator DSP card, the magnetron including the high tension (HT) power supply, and the plasma itself. Also the photo-diode (PD) is shown, as well as the data acquisition card of the PC.

3 Three snapshots from a movie at $10^{5} \mathrm{fps}$. Time between consecutive images is $10 \mu \mathrm{s}$. In the picture of the middle, there is a bright plasma filament along the upper right part of the bulb wall, but this situation does not occur in the preceding (left), nor in the following (right).

Acoustic reflections: the bulb wall is considered as two facing concave spherical mirrors, the furthest and the closest to the source point. . . . . . . . . . . . . . . . . . .

5 (a) AC component of the photo signal (black) and the magnetron command signal (gray) of a plasma out of the resonance. (b) Measurement of the corresponding relaxation times. As the photo signal is in phase with the command signal $\omega_{p}=\omega_{c}=2 \pi 26.1 \times 10^{3} \mathrm{~Hz}$. Therefore $\tau_{e} \approx 0.7 /\left(0.164 \times 10^{6}\right) \mathrm{s} \approx 4 \mu \mathrm{s}$ and, similarly, $\tau_{v}$ ranges from $10 \mu$ s to $30 \mu$ s (typical values) . . . . . . . . . . . . . . . .

6 (a) AC component of the photo signal (black) and the command signal (gray) of a plasma in the spherical resonance. (b) Photo-signal of a spherical resonance plasma (black) and the sum of two sinusoids where one has the frequency of $28.2 \mathrm{kHz}$ and the other has $1 \%$ lower frequency (gray). (c) Normalized spectral power of the photo signal. The horizontal axis is the frequency normalized to the modulation frequency.

7 (a) AC component of the photo signal of two plasmas shown in black and gray. Both plasmas undergo a transition to the spherical resonance at $t=17 \mathrm{~ms}$. (b) The product $\omega_{p} \tau_{e}$ of the two plasmas. (c) The product $\omega_{p} \tau_{v}$ of the two plasmas. Only the values of $\tau_{e}$ and $\tau_{v}$ are shown with $R^{2}>0.9$ of the least squares fit.

8 Heating interruptions, (a) as the plasma is in the spherical resonance, (b) as the plasma is not in the

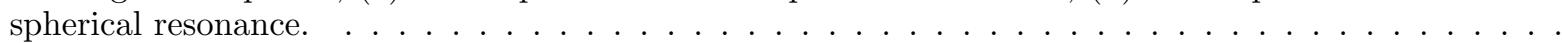

9 The constant of equilibrium $K_{e}$ as a function of temperature. Comparison of (15) - dashed line - and

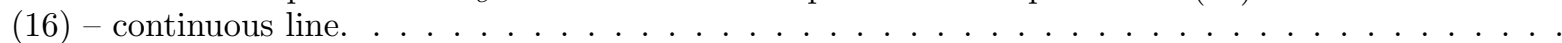

10 The predicted DC degree of dissociation as a function of temperature, for $n_{0}=1.6910^{19} \mathrm{~cm}^{-3} . K_{e}$ is calculated according to $(16) \ldots \ldots \ldots \ldots \ldots \ldots$

11 The frozen (solid line) and the equilibrium (dashed) phase velocity of sound as a function of temperature.

12 Schematic illustration of the time lag of the two echoes. The points $S_{R}$ and $S_{L}$ are the images of $S$ by reflection into the mirrors on the right- and left-hand sides, respectively. . . . . . . . . . . . . . .

13 Spectrum of possible modes of acoustic resonance in a homogeneous isothermal solid sphere. The horizontal axis is the order of the spherical Bessel function $l$. For each order $l$, the set of modes given as the order extremum of the Bessel function $n$ are shown by bars. The vertical axis is the temperature for the mode frequency of $28.2 \mathrm{kHz}$. For the $l=0$ case the first mode is not shown. The white bars are excluded, because their temperatures are too low in regard to the measured bulb surface temperature $\left(900{ }^{\circ} \mathrm{C}\right)$. Among the other bars, there is one pair of almost same height as shown in black. These modes could thus coexist and the temperature would then be of $1930{ }^{\circ} \mathrm{C}$. Therefore, as this is the only configuration that can cause a beat, we conclude that, at the spherical resonance, the acoustic mean of the plasma temperature was about $1930^{\circ} \mathrm{C} \ldots \ldots \ldots \ldots \ldots$

14 Left: The spherical mode $(0,2)$. Right: The non-spherical mode $(3,1)$. The outer sphere is the bulb boundary. Inside is drawn the nodal surface of the pressure, see Appendix C, (77), (83), (84), (86). . .

15 Radial profile of the dimensionless pressure in the normal mode $(l=0, n=2)$, see Appendix C, (77),

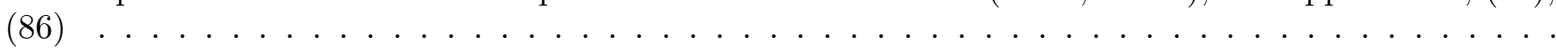

16 Ratio of the frequency of the plasma response to the modulation frequency $f_{p} / f_{c}$ of a plasma in a spherical resonance. . . . . . . . . . . . . . . . . . . . . . . . . .

17 The normalized amplitude vs the ratio of the frequency of the plasma response to the modulation frequency $f_{p} / f_{c}$ in a spherical resonance. . . . . . . . . . . . . . . . . . . . . . . . . .

18 The normalized amplitude vs the phase advance of the microwave pulse in respect to the plasma oscillation. 39

19 (a) AC component of the photo signal of a plasma going undergoing a period of spherical resonance.

(b) Phase shift of the microwave pulse in respect to the plasma oscillation. . . . . . . . . . . . .

20 Dispersion curve: Phase velocity of sound versus the non-dimensional characteristic relaxation time, according to (11) and (26) and, at $523 \mathrm{kPa}($ see Table 1$) \ldots \ldots \ldots$

21 Complex isentropic coefficient versus the non-dimensional characteristic relaxation time, according to (11), at $523 \mathrm{kPa}$ (see Table 1). (a) Real part. (b) Phase angle. . . . . . . . . . . . . . . . . . 
23 Normalized spectrum of the photon flux. Emission of the plasma (continuous line) and of the black-body (dashed lines) at the plasma temperature expected as an average throughout the bulb volume, $2200 \mathrm{~K}$, and in the center, $4100 \mathrm{~K}$ (see Sect. 6.2). The background color shows in white the spectral band of the visible. Note: the peak is due to a trace of Lithium that has degased from the bulb glass (additive of the manufacturer) $[52] \ldots \ldots \ldots \ldots \ldots \ldots \ldots$ 


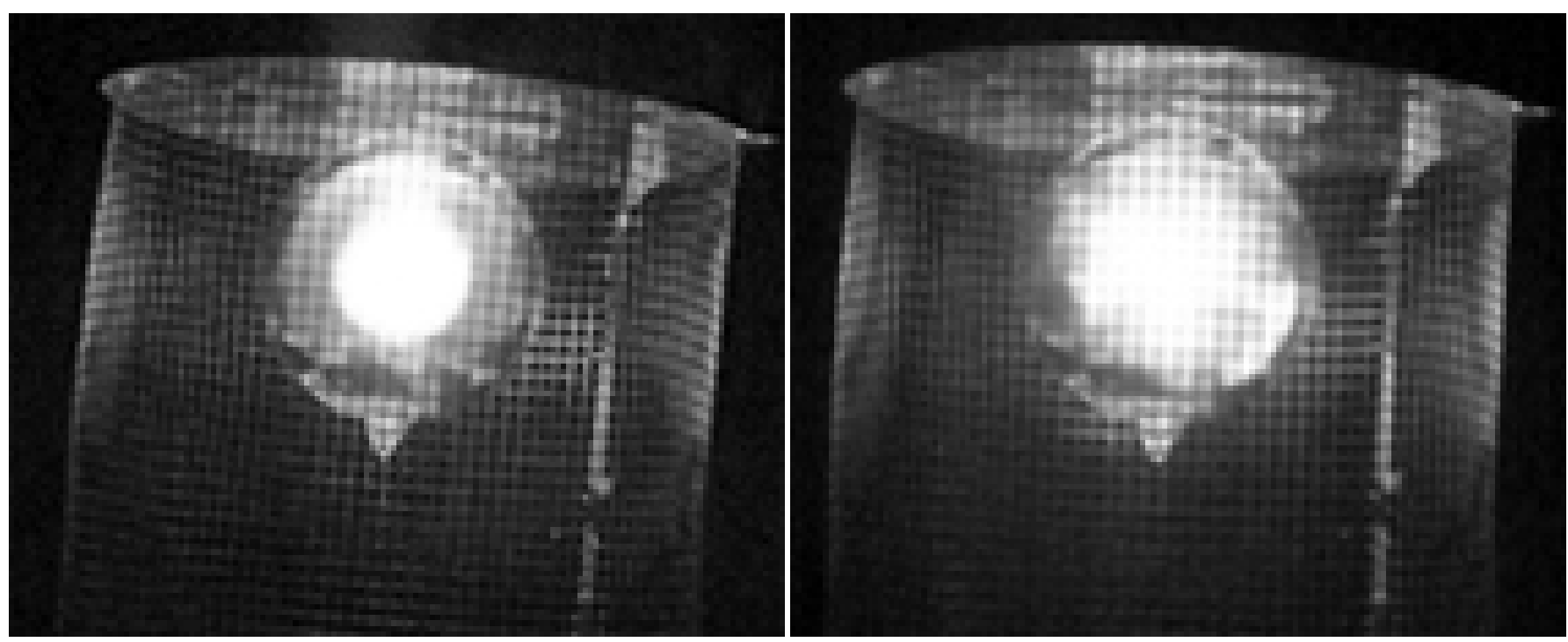

Fig. 1 


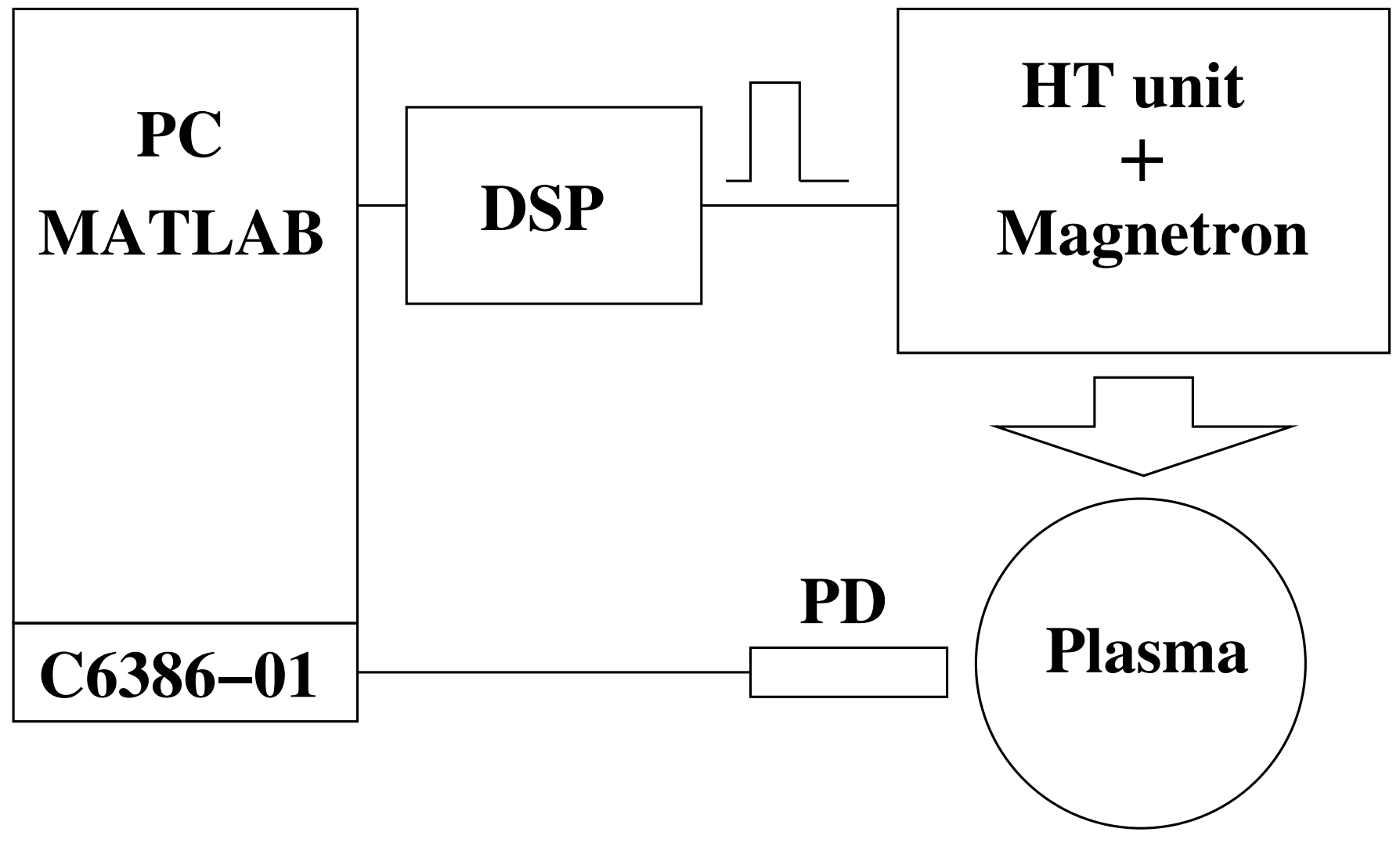

Fig. 2 


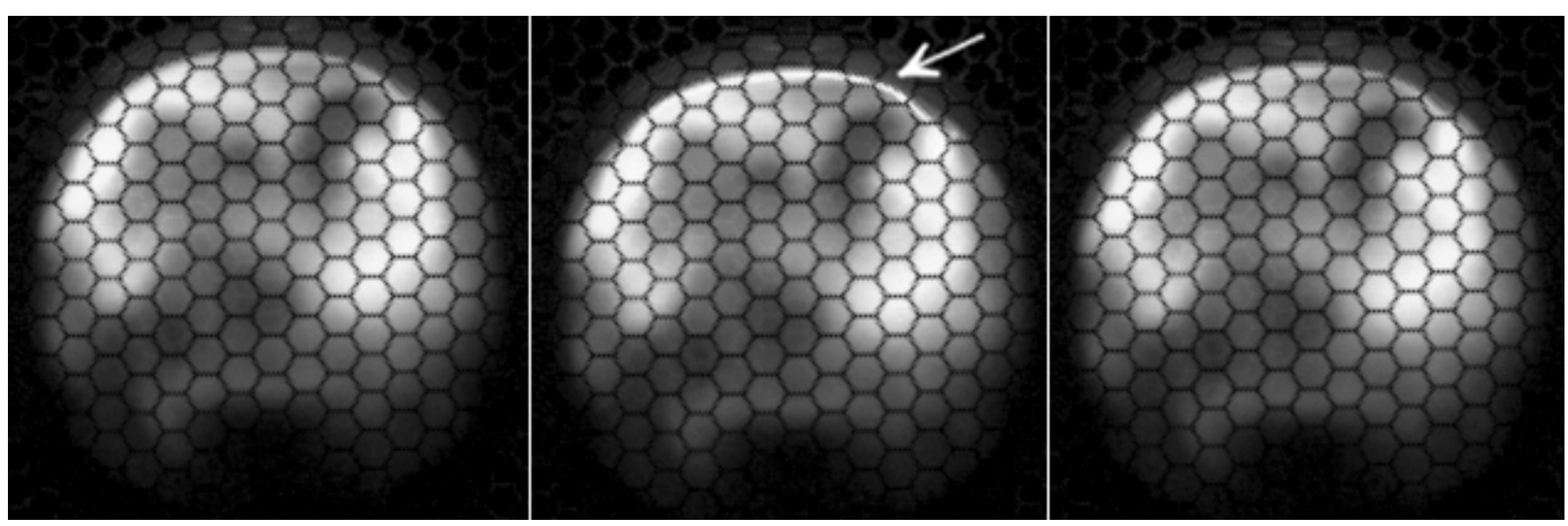

Fig. 3 


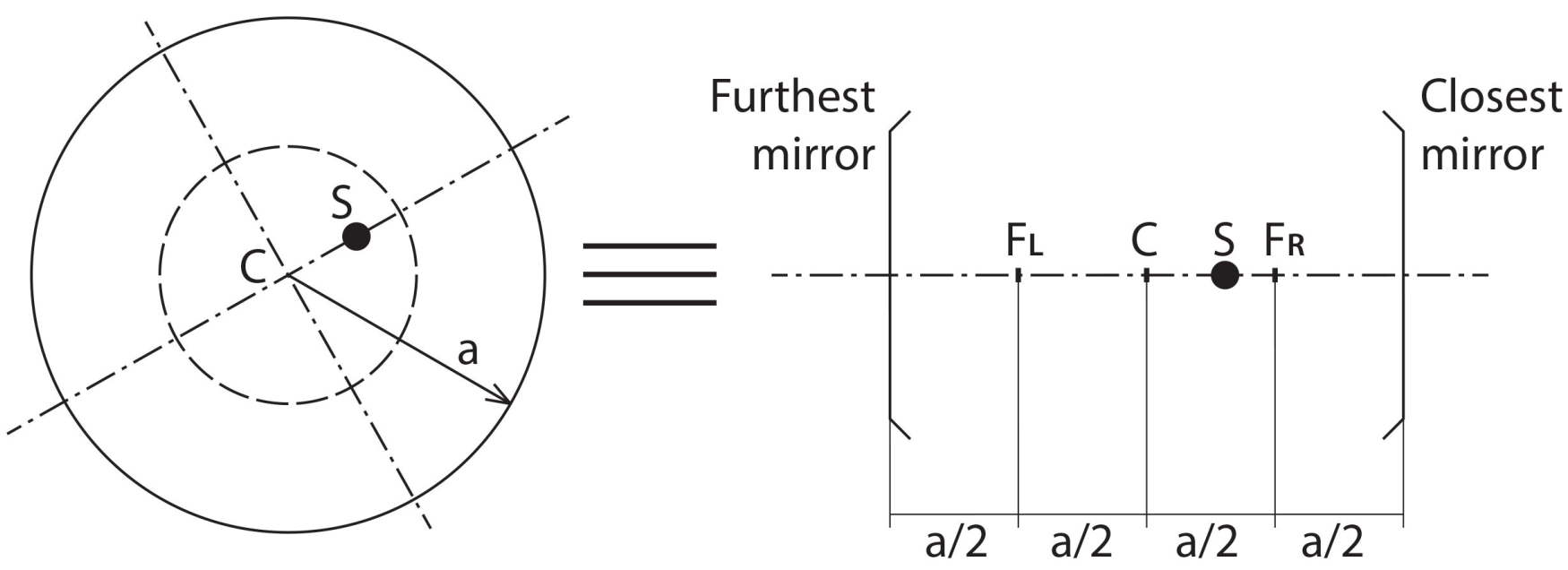

Fig. 4 

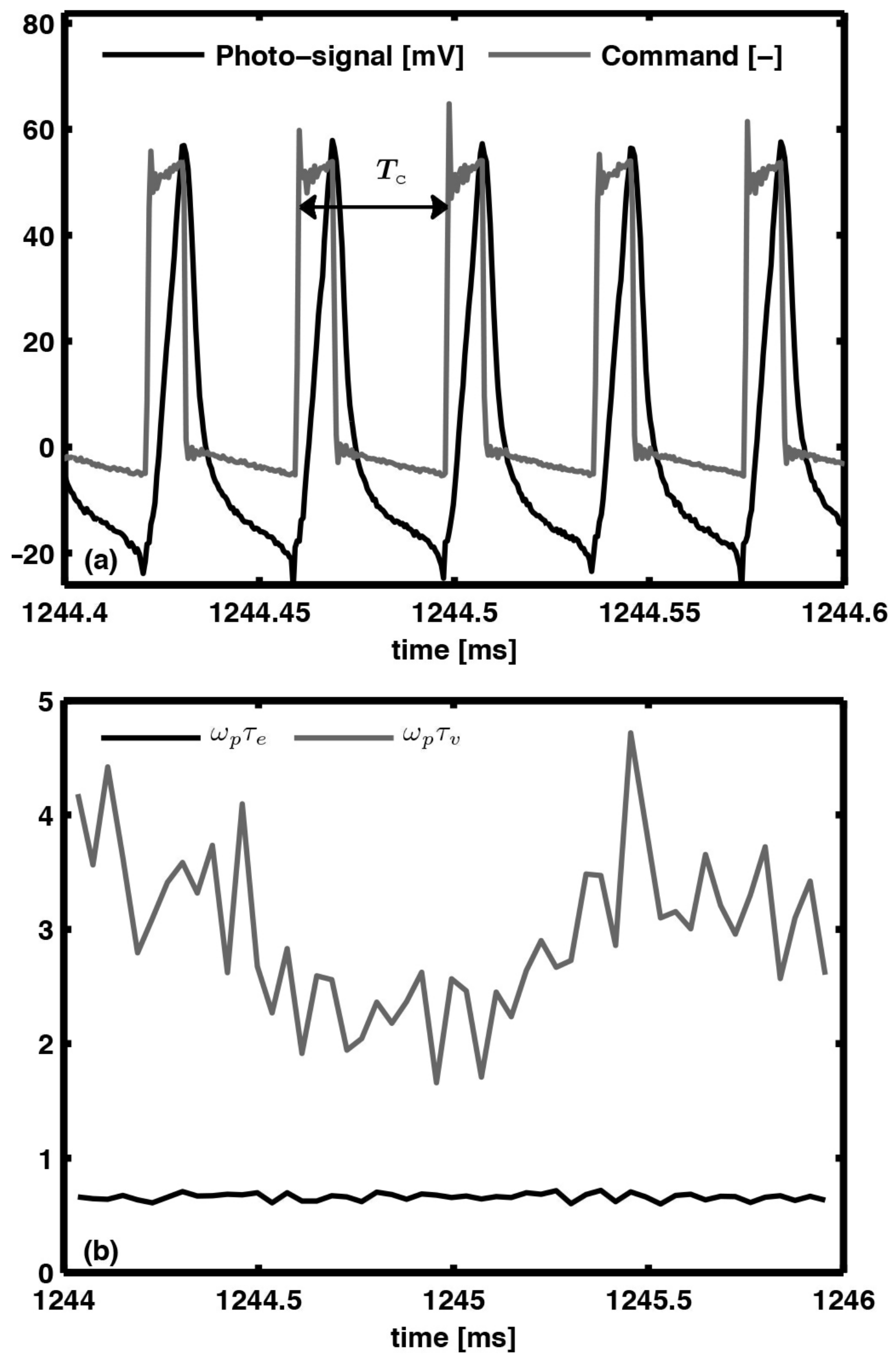

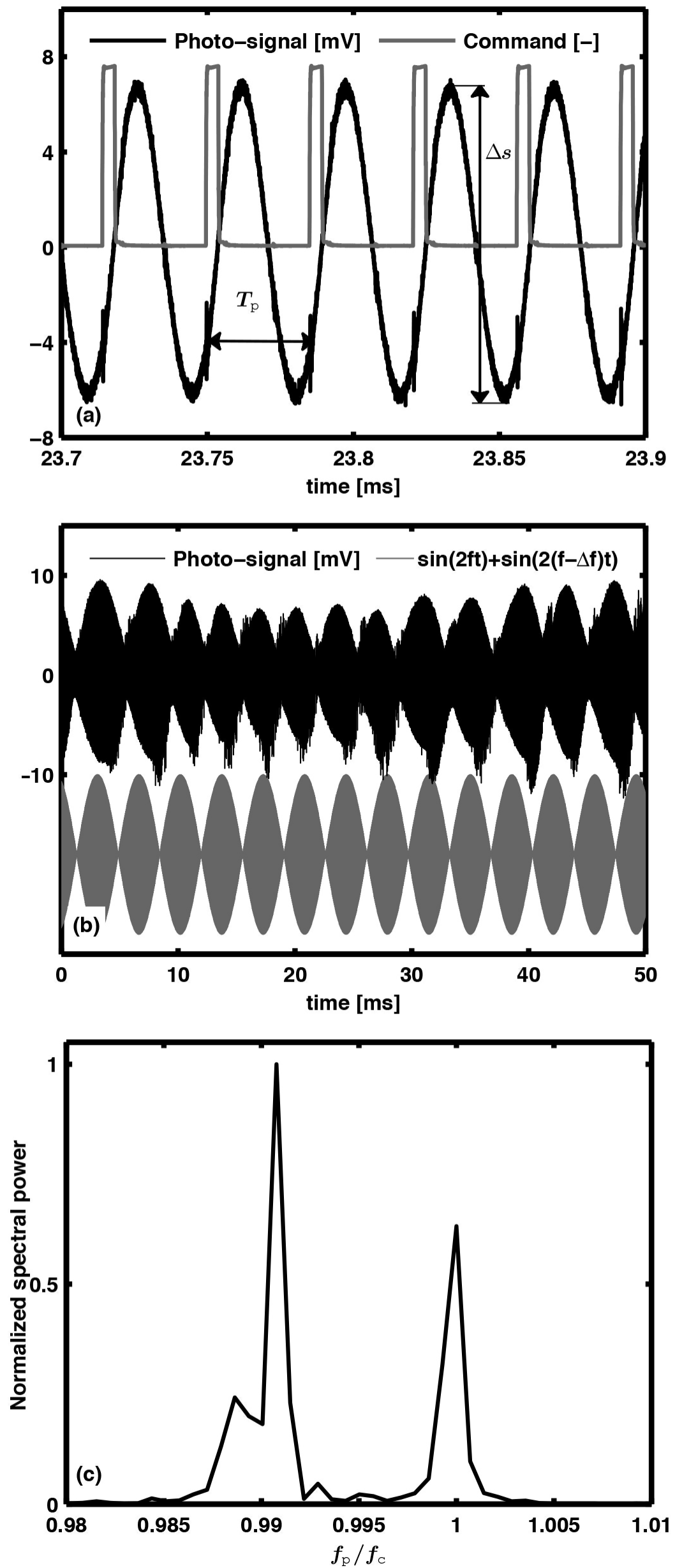

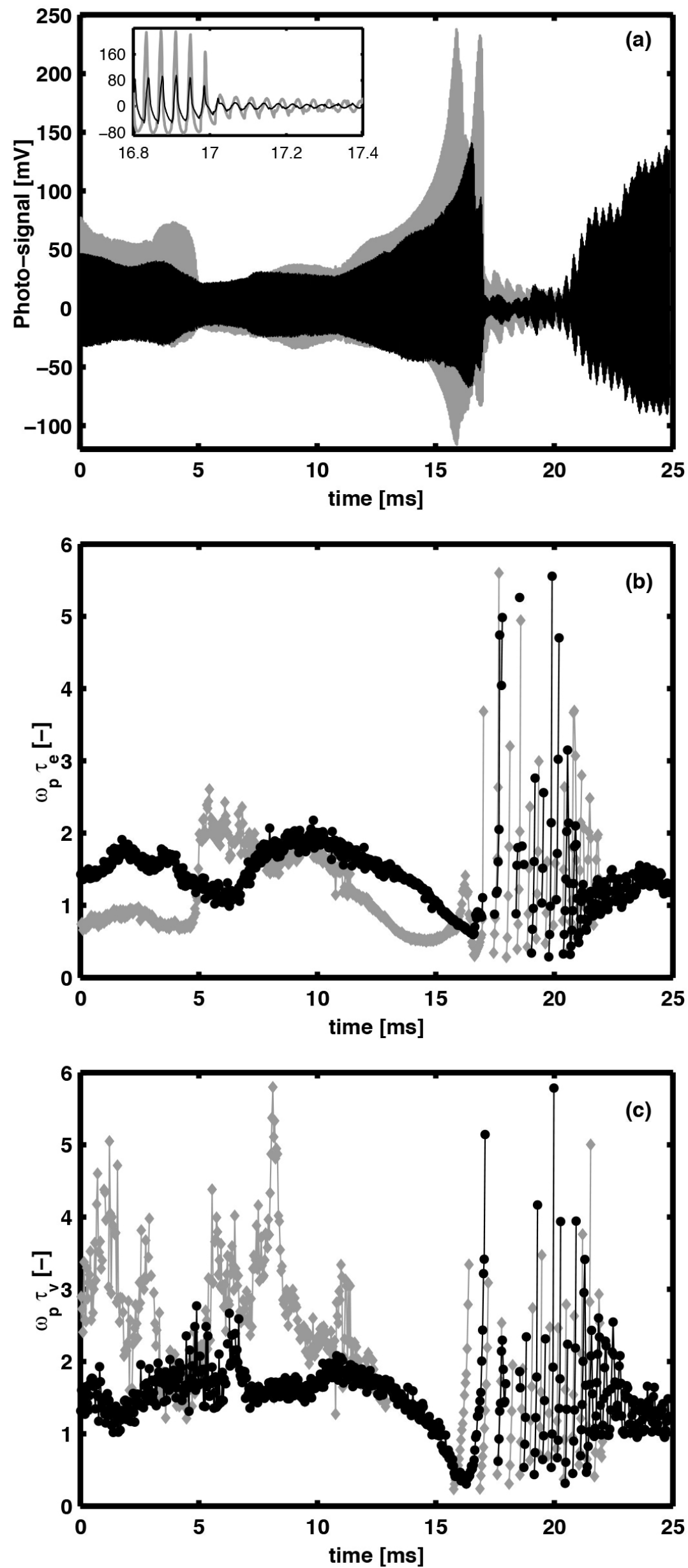

Fig. 7 

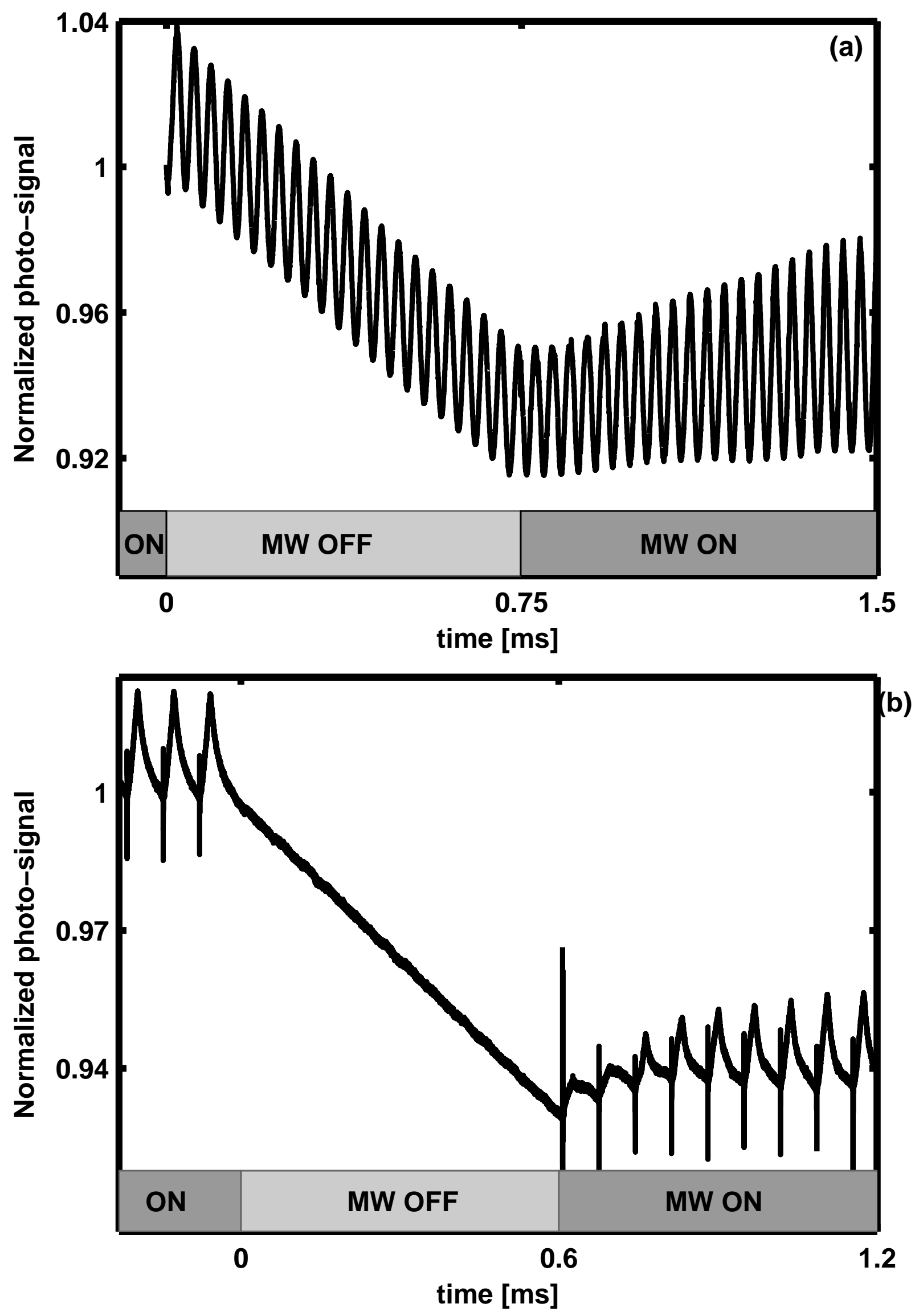

Fig. 8 


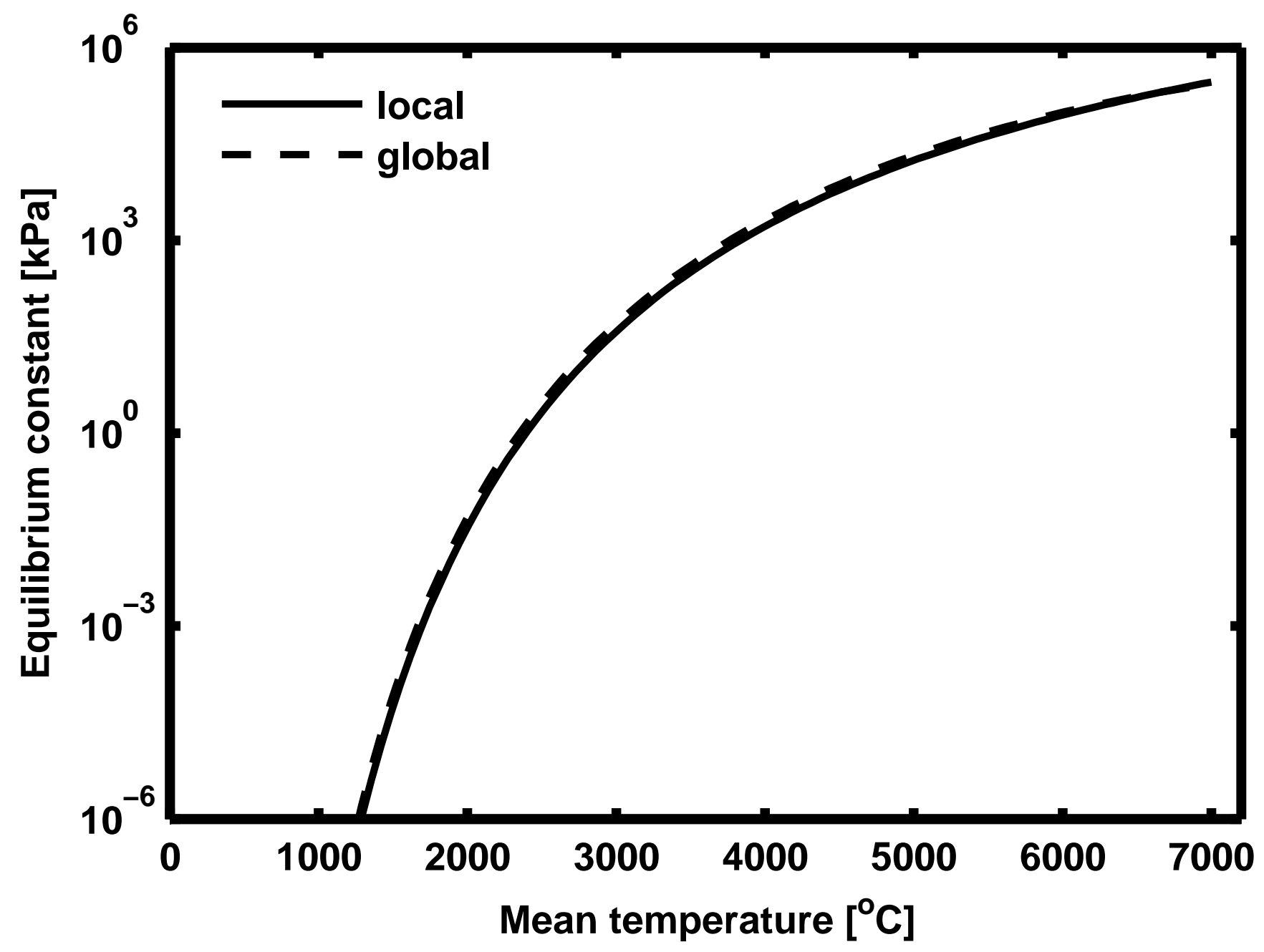

Fig. 9 


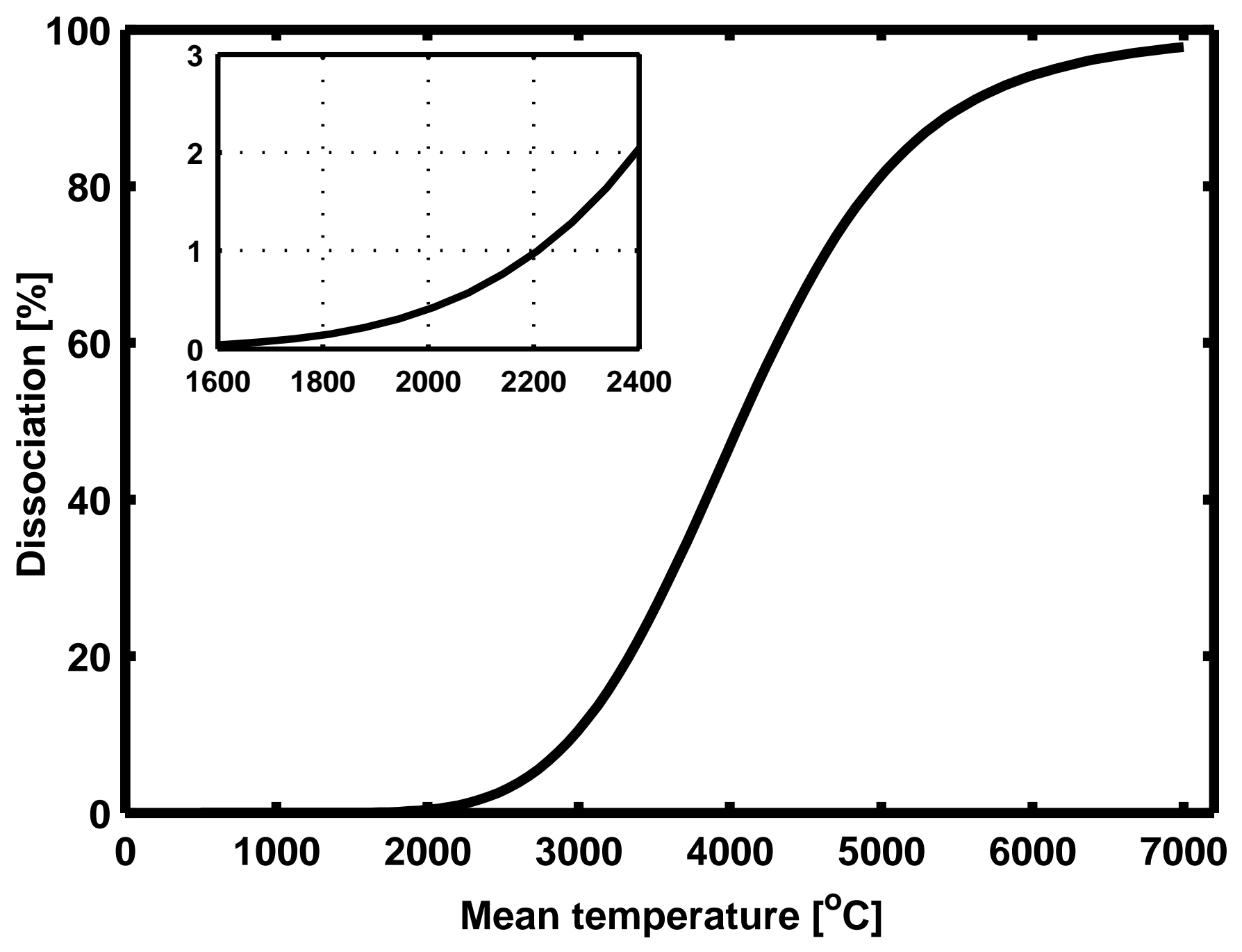

Fig. 10 


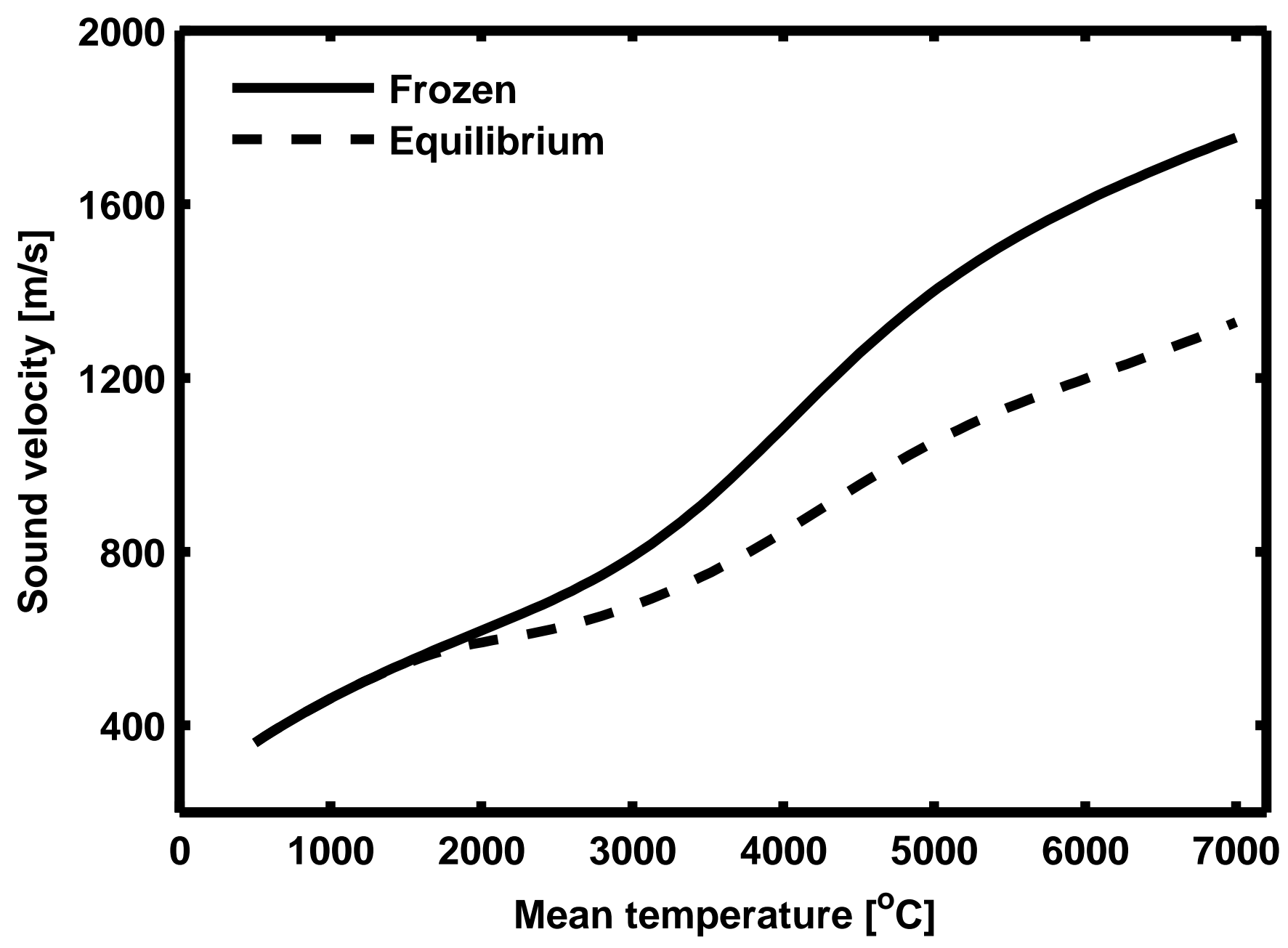

Fig. 11 
time

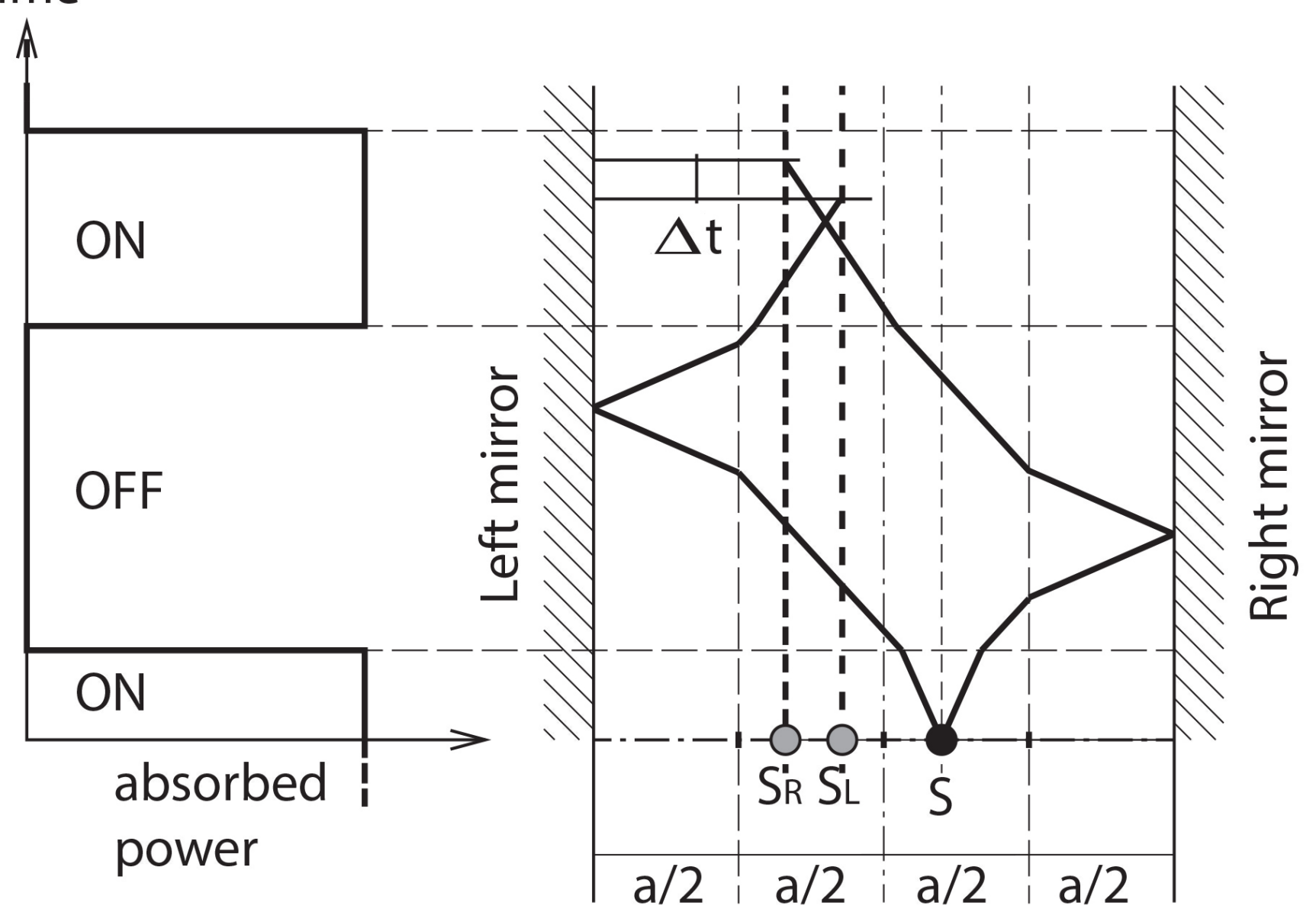

Fig. 12 


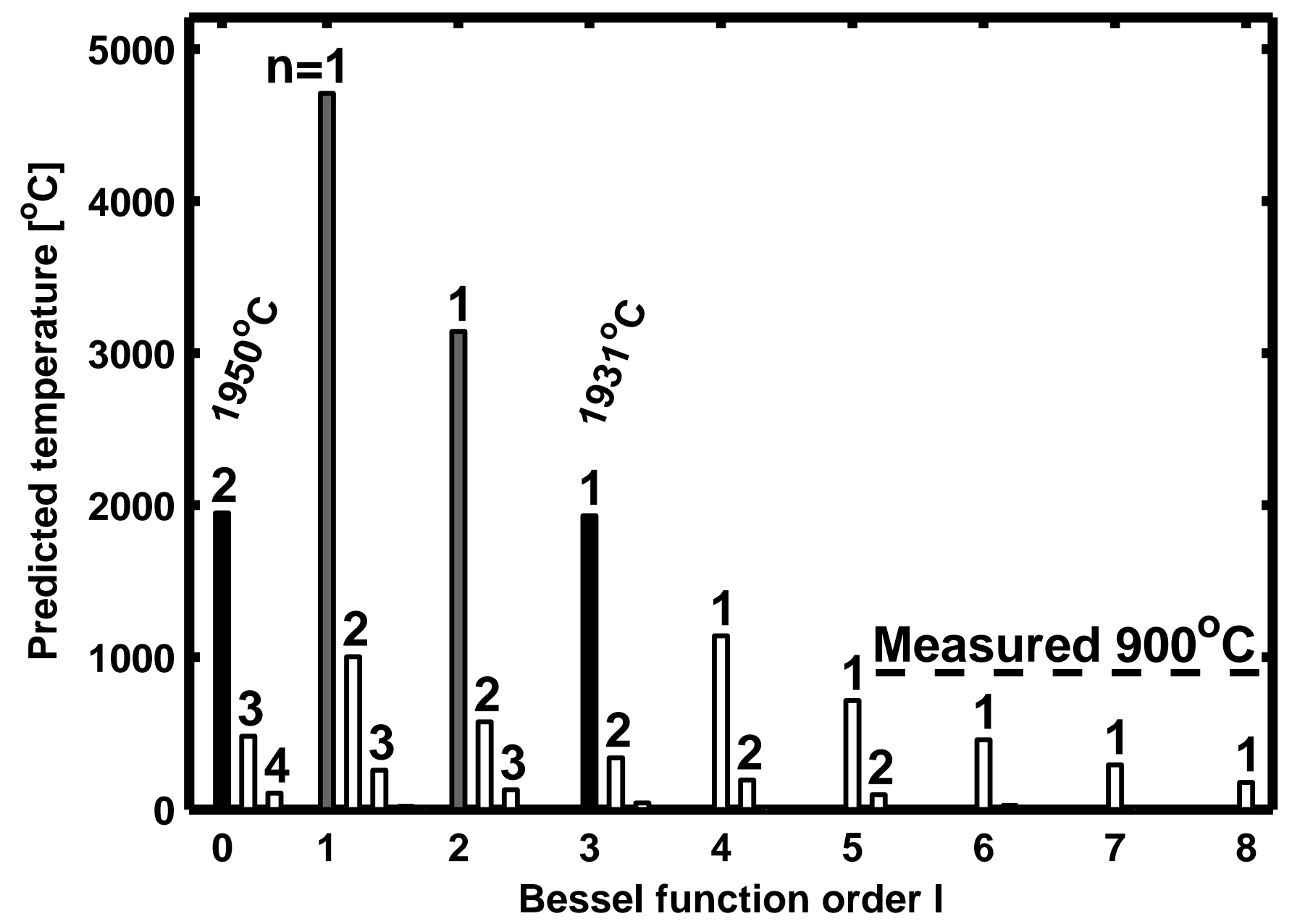

Fig. 13 
FIGURES

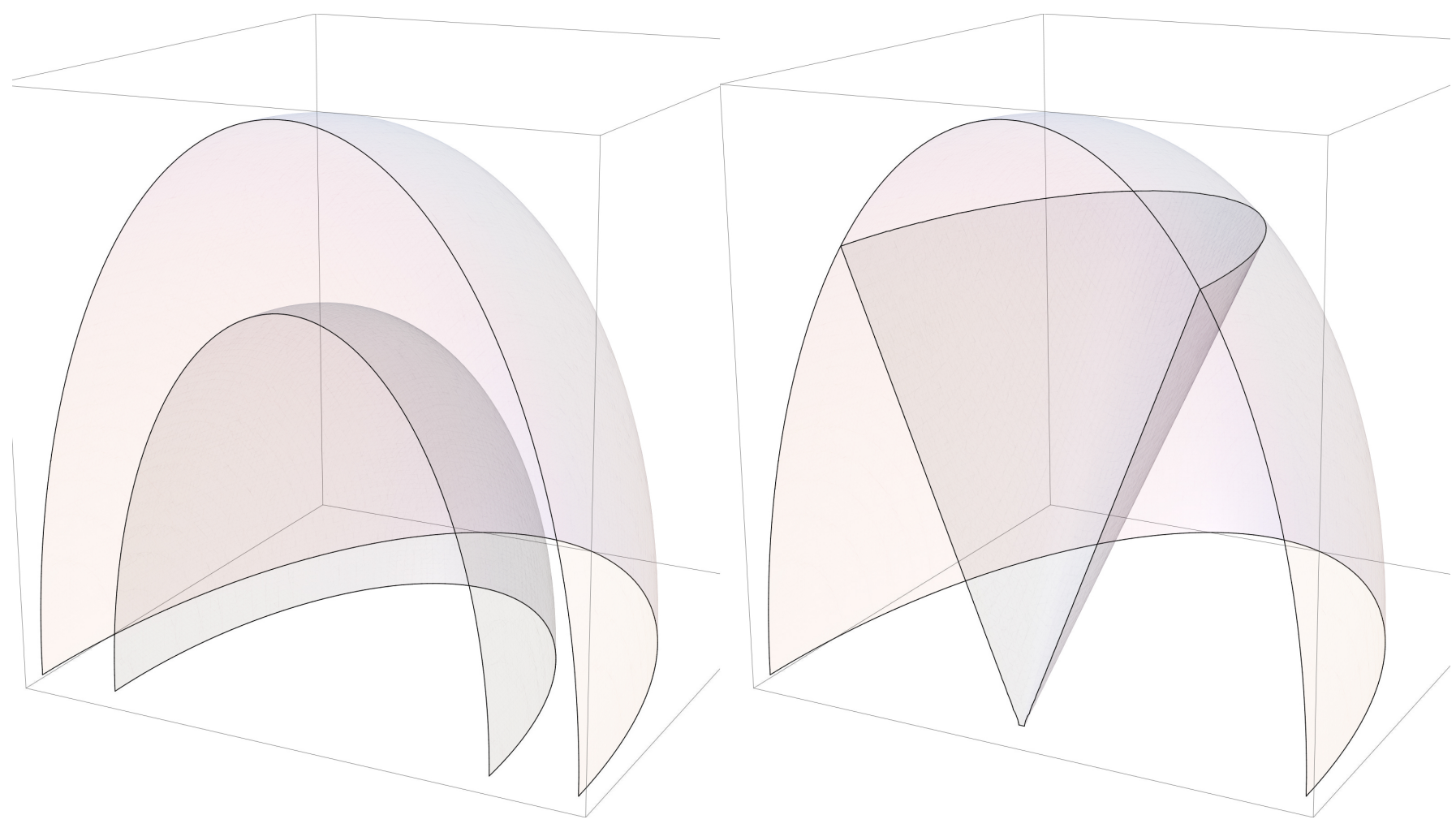

Fig. 14 


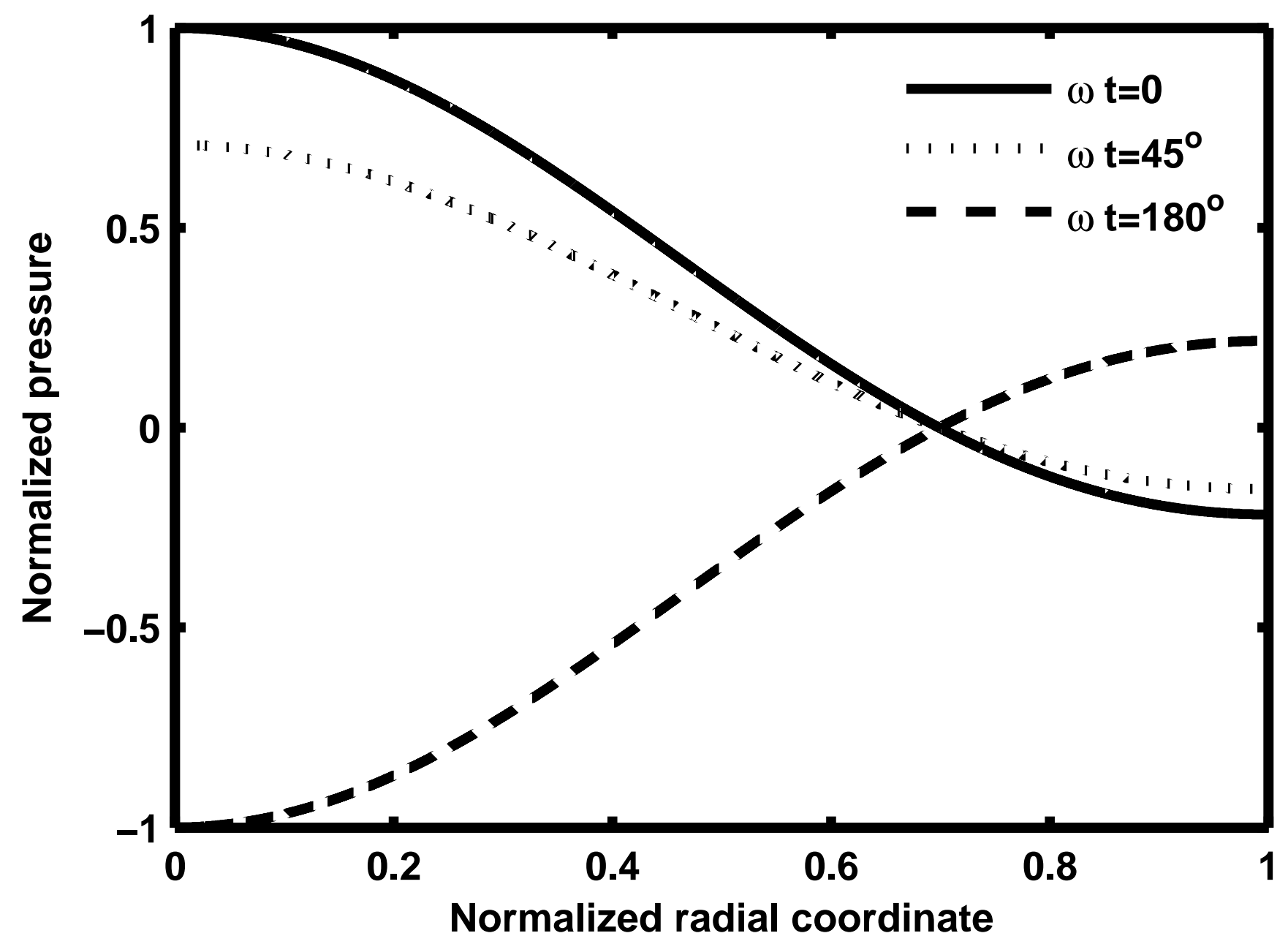

Fig. 15 


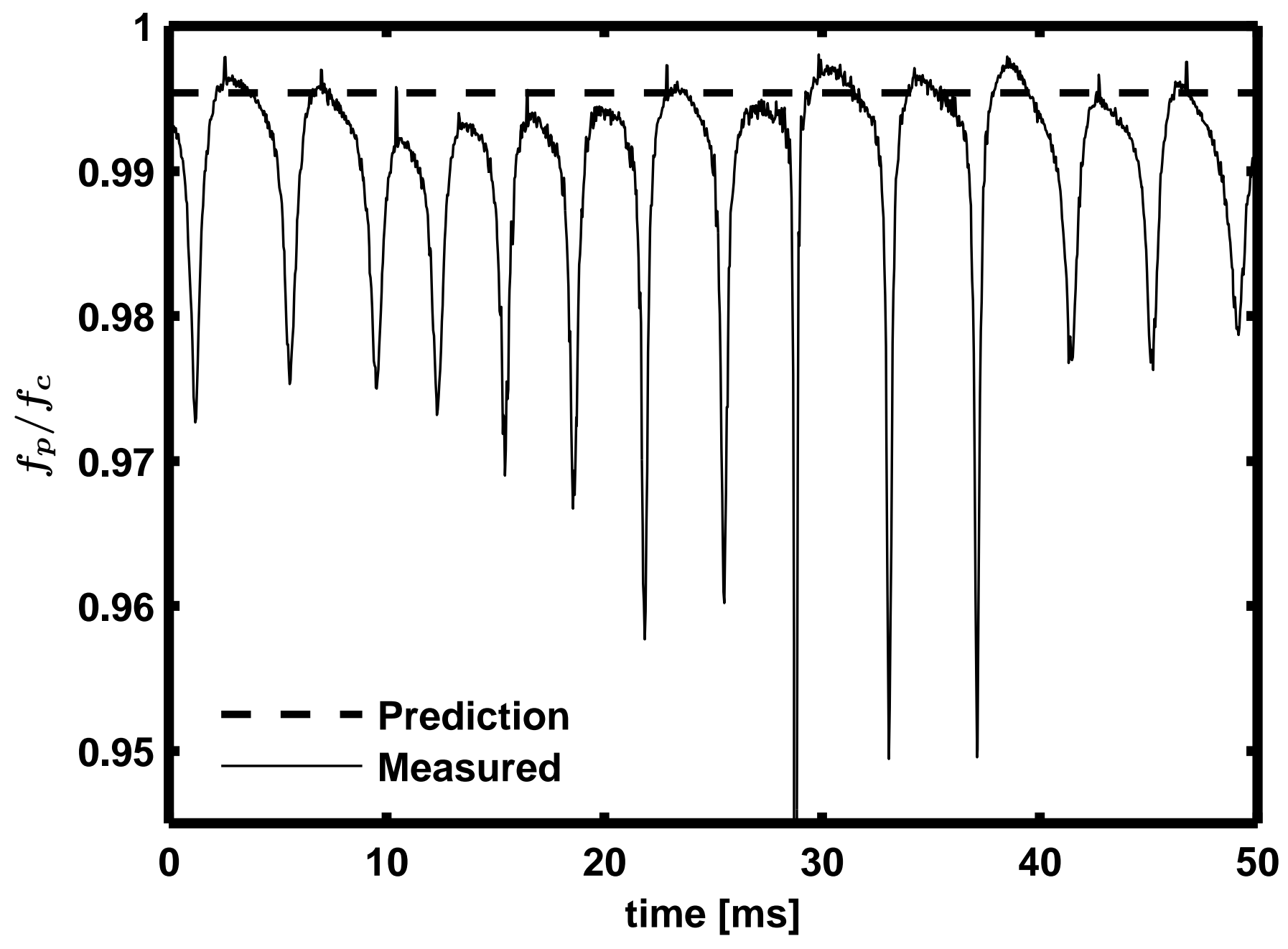

Fig. 16 


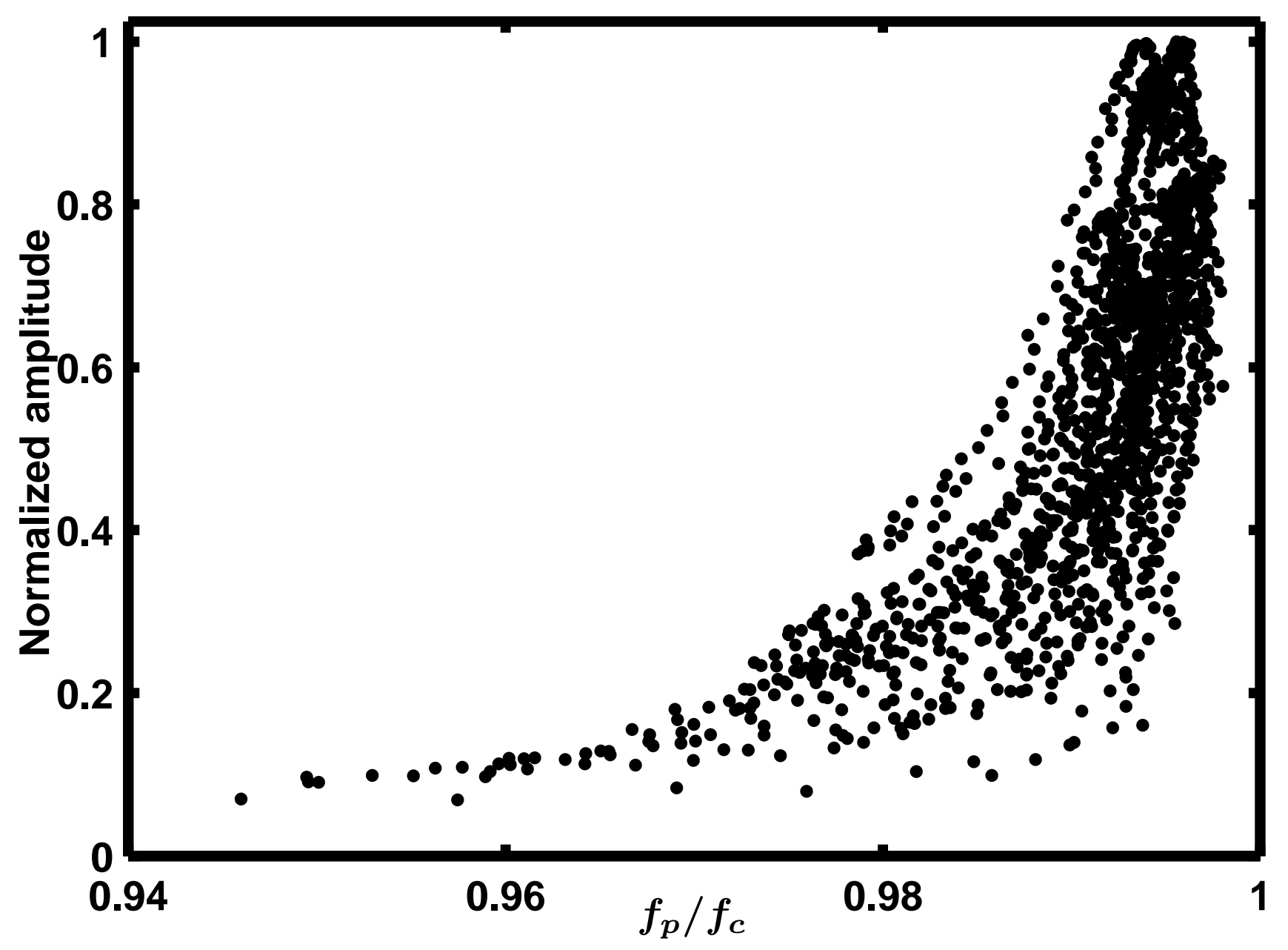

Fig. 17 


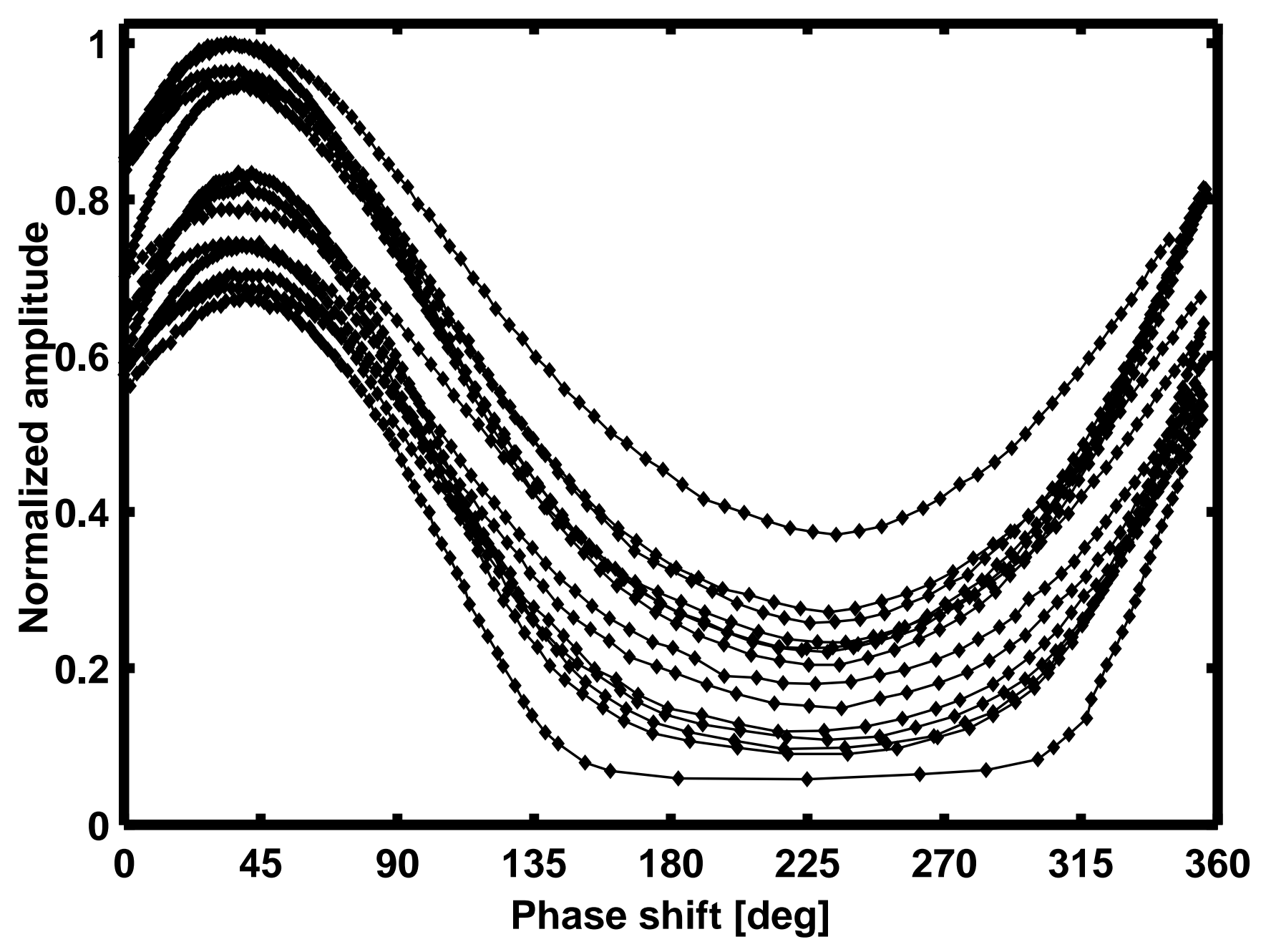

Fig. 18 


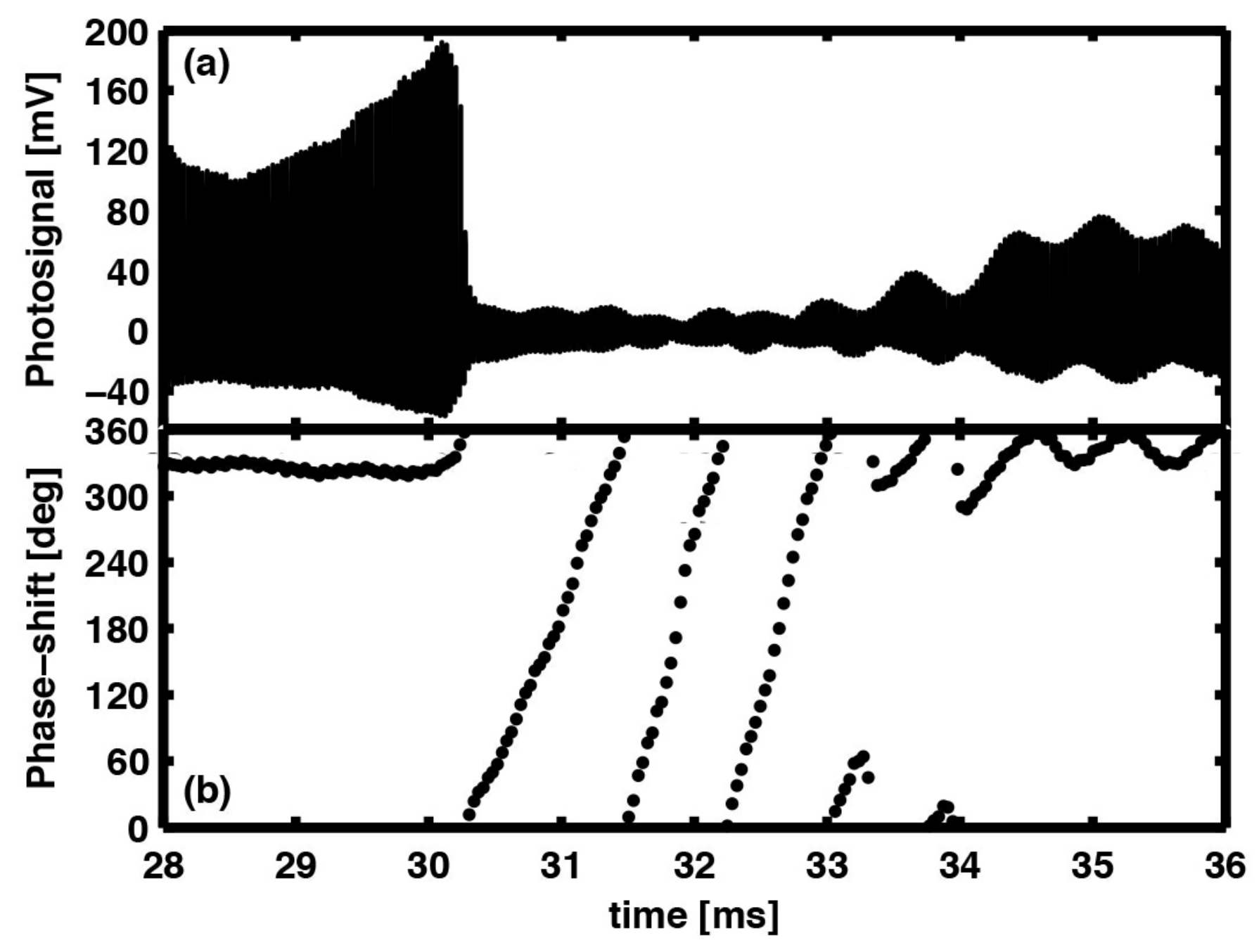

Fig. 19 


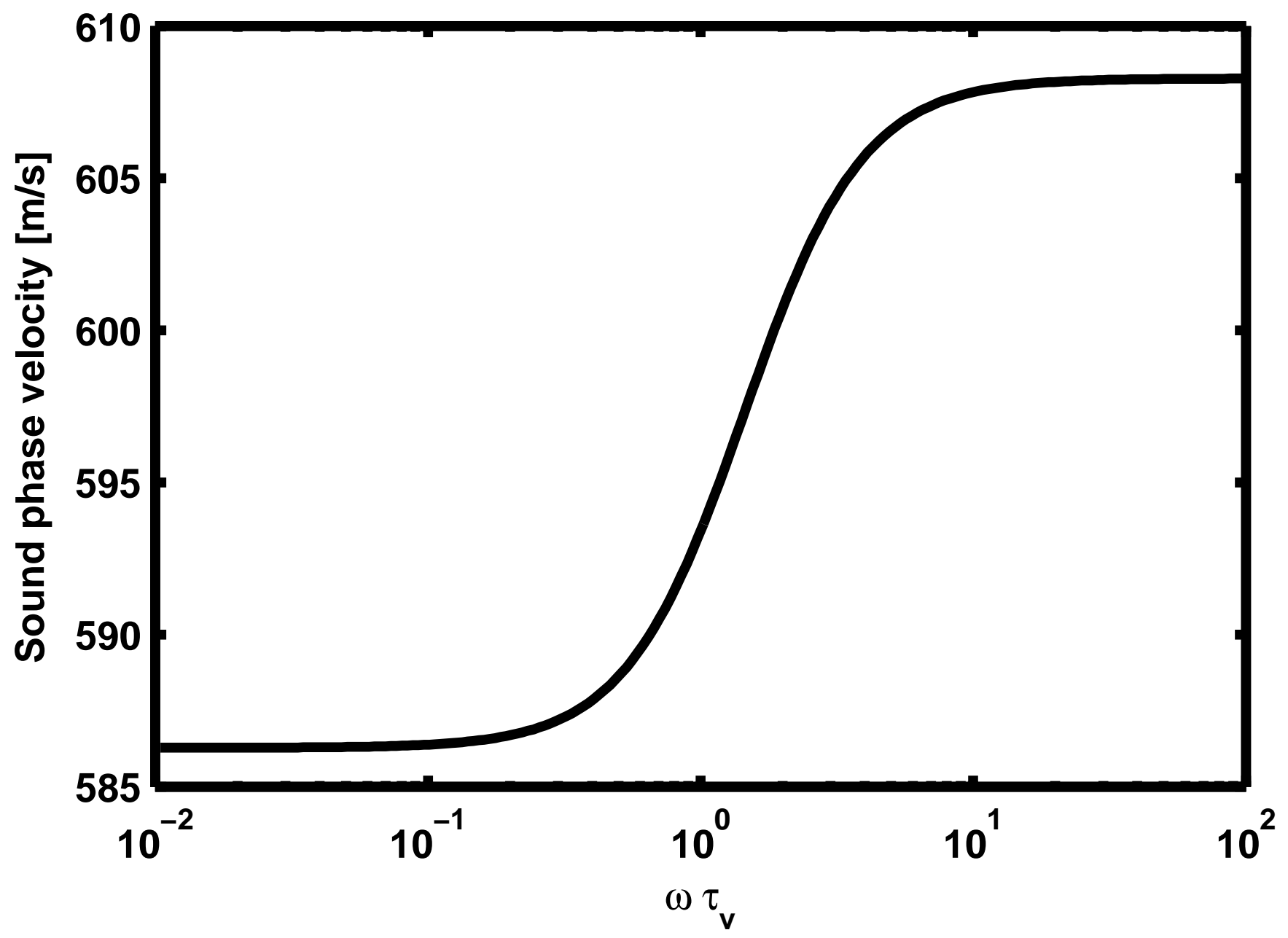

Fig. 20 

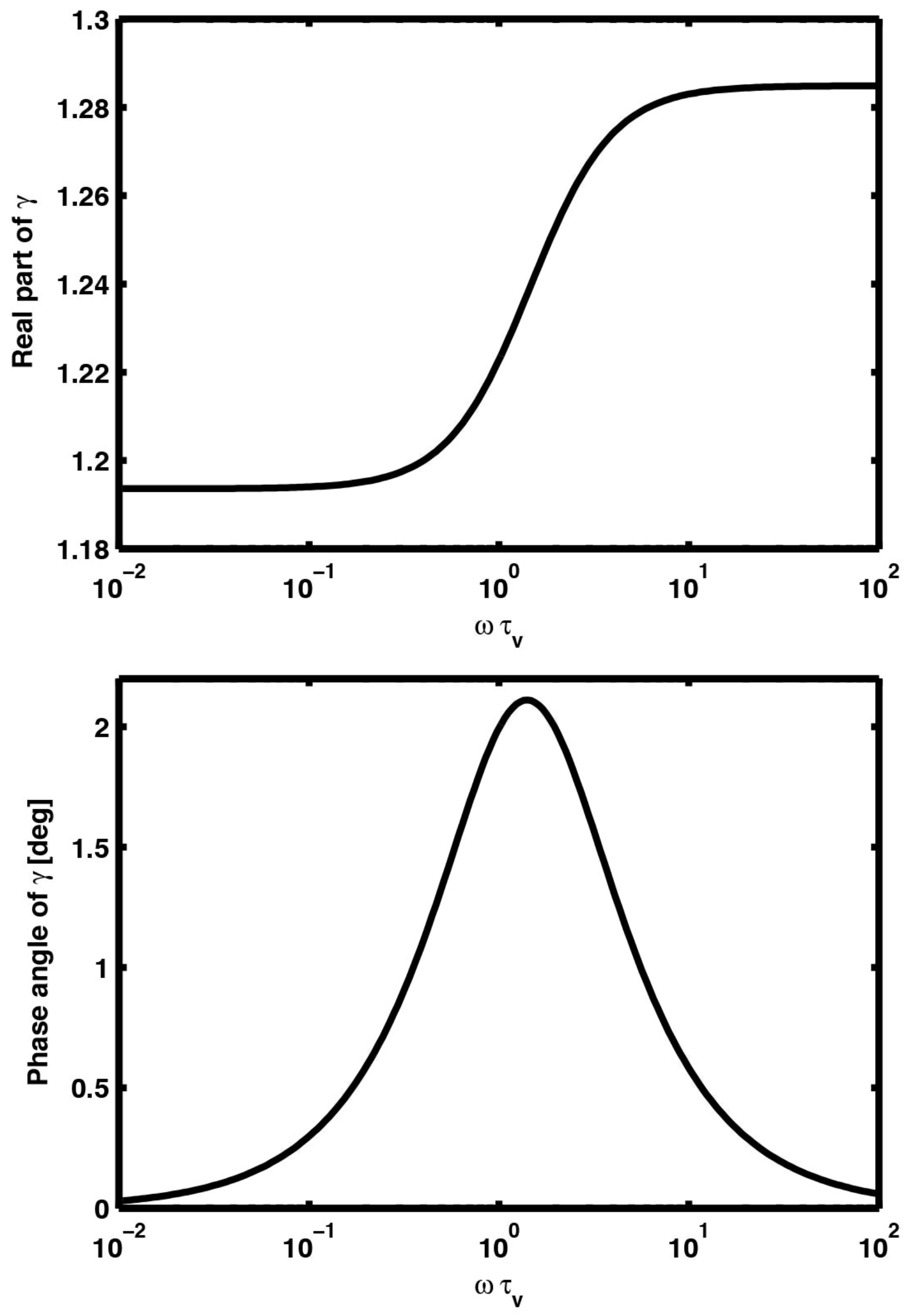

Fig. 21 
Population number

Energy (in eV)

of disulfur

$\mathrm{N}_{1} / 2$

(4.5)

个

$\mathrm{N}_{2}{ }^{*}$

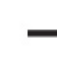

$\mathrm{N}_{2}{ }^{0}$

$\mathrm{h} v_{\mathrm{m}}(2.2)$

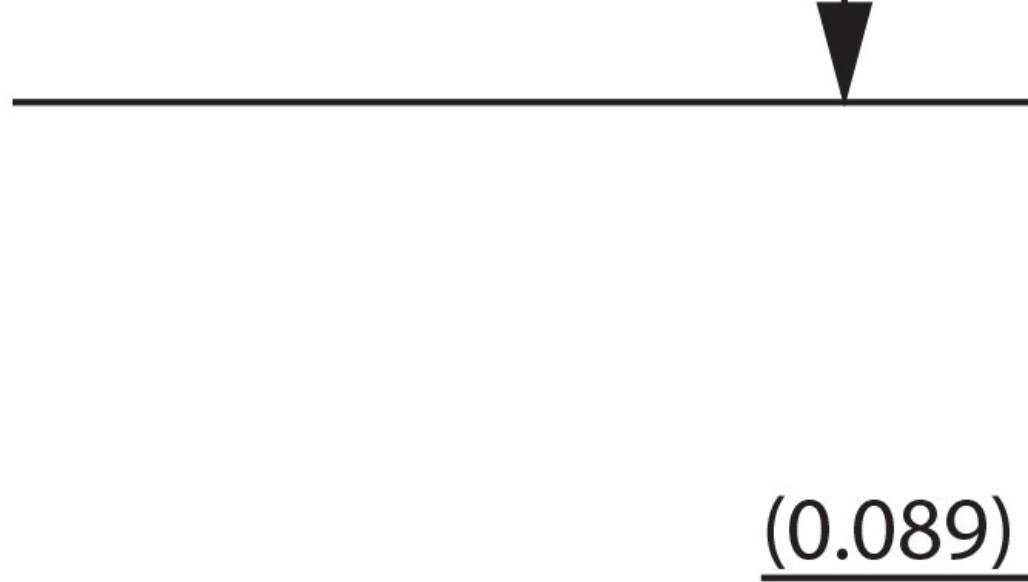

$E_{v b}$

$h v_{m}+E_{v b}$

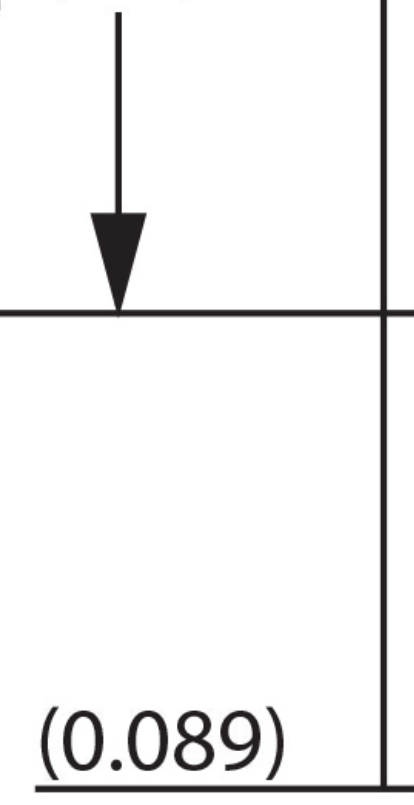

GND

Fig. 22 


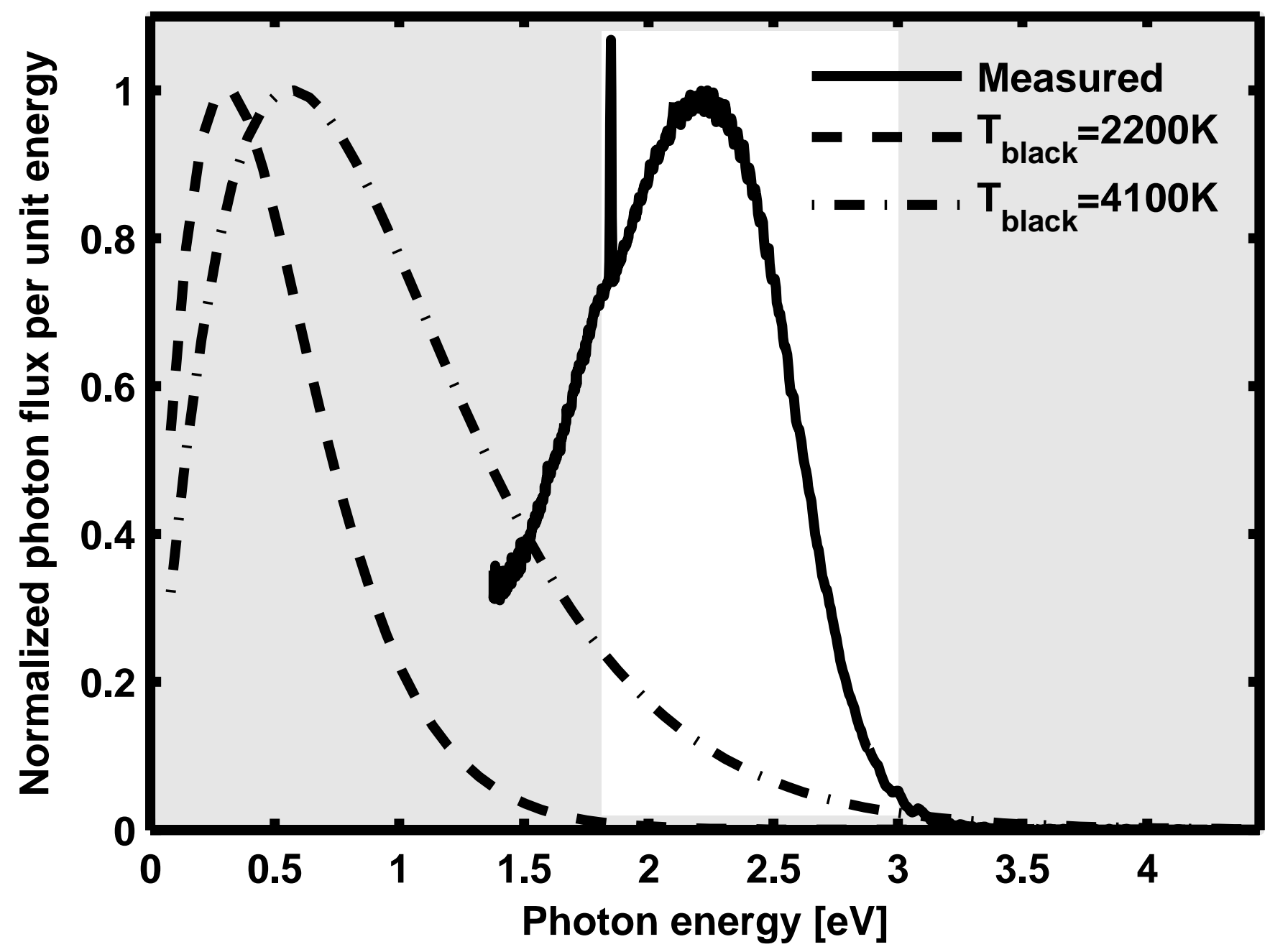

Fig. 23 


\section{List of Tables}

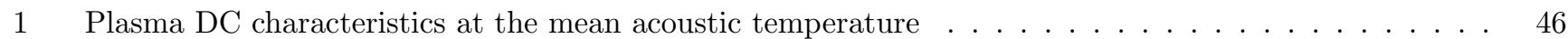

2 The first values of $\chi_{l, n}$ for $l=1, \ldots, 4$ and $n=1,2,3 \ldots \ldots \ldots \ldots \ldots \ldots \ldots \ldots$ 
Table 1

\begin{tabular}{lccc}
\hline name & symbol & value & unit \\
\hline mean acoustic temperature & $T_{m}$ & 1930 & ${ }^{\circ} \mathrm{C}$ \\
pressure & $\mathrm{p}$ & 523 & $\mathrm{kPa}$ \\
equil. constant of dissociation & $K_{e}$ & 16.6 & $\mathrm{~Pa}$ \\
degree of dissociation & $\alpha$ & 0.28 & $\%$ \\
frozen isochoric heat capa. & $c_{\infty}$ & 55.2 & $\mathrm{~mol} / \mathrm{kg}$ \\
equil. isochoric heat capa. & $c_{0}$ & 78.0 & $\mathrm{~mol} / \mathrm{kg}$ \\
eff. equili. isochoric heat capa. & $c_{0}^{\star}$ & 80.5 & $\mathrm{~mol} / \mathrm{kg}$ \\
frozen isentropic coefficient & $\gamma_{\infty}$ & 1.28 & \\
equil. isentropic coefficient & $\gamma_{0}$ & 1.19 & \\
molar mass & $\bar{M}$ & 63.6 & $\mathrm{~g} / \mathrm{mol}$ \\
frozen phase velocity of sound & $v_{\infty}$ & 608 & $\mathrm{~m} / \mathrm{s}$ \\
equil. phase velocity of sound & $v_{0}$ & 586 & $\mathrm{~m} / \mathrm{s}$ \\
\hline
\end{tabular}


Table 2

\begin{tabular}{cccc}
\hline$l$ & $n=1$ & $n=2$ & $n=3$ \\
\hline 0 & - & 4.49341 & 7.72524 \\
1 & 2.08158 & 5.94037 & 9.20584 \\
2 & 3.34209 & 7.28993 & 10.61384 \\
3 & 4.51410 & 8.58375 & 11.97272 \\
4 & 5.64670 & 9.84045 & 13.29556 \\
\hline
\end{tabular}

